\title{
Roller Bearing Health Monitoring \\ Using CPLE Frequency Analysis Method
}

\author{
Dr. Jen-Yi Jong, Jess H Jones \\ AI Signal Research, Inc., \\ Huntsville, AL 35805
}

\author{
Tony R. Fiorucci \\ NASA/Marshall Space Flight Center, \\ Marshall Space Flight Center, AL 35812
}

\begin{abstract}
This paper describes a unique vibration signature analysis technique - Coherence Phase Line Enhancer (CPLE) Frequency Analysis - for roller bearing health monitoring. Defects of roller bearing (e.g. wear, foreign debris, crack in bearing supporting structure, etc.) can cause small bearing characteristic frequency shifts due to minor changes in bearing geometry. Such frequency shifts are often too small to detect by the conventional Power Spectral Density (PSD) due to its frequency bandwidth limitation. This Coherent Phase Line Enhancer technology has been evolving over the last few years and has culminated in the introduction of a new and novel frequency spectrum which is fully described in this paper.
\end{abstract}

This CPLE approach incorporates phase information into the frequency estimation process. This method first performs regular Order Sampling (OS) pre-processing using a once/revolution (1/Rev) shaft speed key phasor measurement as a reference signal. The resulting OS signal is then transformed into the frequency domain using the conventional Fast Fourier Transform (FFT). The signal component at each FFT discrete frequency (spectral line) is further transformed into a new CPLE frequency domain. This second transformation is in the ensemble direction, across multiple FFT blocks, and it preserves the both amplitude and phase information contained in the original OS time signal. This transformation effectively decomposes the signal power at each spectral line into finer CPLE frequency bands corresponding to plus/minus half-bandwidth around each spectral line. Due to a unique coherent phase relationship associated with a speed-related signal component (e.g. bearing-related signal, gear mesh signal, Synchronous ( $\mathrm{N}$ or sync) harmonics and sub-harmonics), this speed-related component's power distribution will concentrate around a maximum peak within the CPLE frequency band. Otherwise, its power distribution will be dispersed for all non-speed-related signals. As a result, two functions, CPLE-PSD and CPLE-Coherence can be defined [Reference 1, 2]. CPLEPSD, which is defined as the signal power of the maximum peak, can provide significant spectral enhancement capability of the RPM-related signal in the presence of high background noise. This will improve the signal-to-noise ratio of the critical bearing related signals. CPLE-Coherence, which is defined as the ratio of power between the maximum peak and the composite (i.e. total energy) over the entire CPLE frequency band, can effectively measure the degree of non-linear correlation between signal components at any frequency with respect to the rotating machinery speed (RPM). This 
paper focuses on a "third" new function, the CPLE-Frequency, which is defined as the location of the maximum peak within the CPLE-frequency band. CPLE-Frequency can significantly improve the bandwidth resolution so that small frequency shifts of speedrelated signal components can be detected.

For rotating machinery applications, CPLE analysis requires a 1/Rev key phasor signal to perform OS pre-processing. However, in many situations, these key phasor signals may not be available. NASA's Space Shuttle Main Engine (SSME) is one such example. During flight operation, the speed sensor measurements for the engine turbopumps are not recorded with the related vibration accelerometers. Even during ground testing, the speed sensor for the SSME High Pressure Oxygen Turbopump (HPOTP) is not recorded. The omission of these speed measurements is usually due to safety concerns related to the key phasor's intrusive installation (e.g. in a high pressure liquid Oxygen environment for the HPOTP). As a result, the ability/reliability for health monitoring and post-test diagnostic evaluation is severely limited. In this case, the CPLE analysis approach can still be achieved using a novel Pseudo Key Phasor (PKP) technique to reconstruct a $1 /$ Rev PKP signal directly from external vibration measurements. This also enables other powerful signal analyses techniques (such as Time Synchronous Averaging) that require a key phasor to become applicable, and consequently enhances fault detection and diagnostic capabilities. Accordingly, this will enable a "non-intrusive vibration monitoring system" (NI-VMS) to be developed for rotating machinery (e.g. rocket/aircraft engine, power-plant/production-line machinery, etc.) when intrusive installation of a key phasor is not available/feasible due to safety/cost concerns.

A baseline study was performed to investigate the bearing frequency characteristics of the High Pressure Fuel Turbopump (HPFTP) Turbine End Roller Bearing (TERB) from SSME static-test history which includes 246 SSME ground tests and flights. Figure 1 shows a typical CPLE spectral analysis (CPLE PSD and CPLE-COH) result from a SSME ground test 902-861 (HPFTP Unit 8212R1). Since a 4/Rev speed measurement is available for ground test of this unit, Order Sampling pre-processing was first performed on all accelerometer measurements using the speed probe as the reference signal. Figure1(a) shows the resulting OS PSD of a Turbine-End accelerometer. (Note: The $\mathrm{x}$-axis of the OS PSD is usually plotted as order frequency. However, the $\mathrm{x}$-axis in figure 1 is plotted as OS frequency which equates to Order frequency multiplied by a normalized Sync frequency of $512 \mathrm{~Hz}$ in this case). Subsequently, CPLE spectral analysis was performed on the OS signal to generate CPLE-PSD \& CPLE-COH as shown in figure1(b) and (c). Compared to the OS PSD, the overall noise floor of the CPLE-PSD dropped about $12 \mathrm{~dB}$. As a result, several RPM-related signal components were enhanced (such as sync harmonics at $4 \mathrm{~N}$ and $7 \mathrm{~N}$ ). In particular, a couple of bearing signatures denoted "C" \& "I-2N" also emerge from the noise floor, which represent, respectively, Cage frequency $(\mathrm{C})$ of the TERB and the turbine end roller bearing Inner Roller Pass (IRP) frequency modulated with $2 \mathrm{~N}(\mathrm{I}-2 \mathrm{~N})$. These two bearing signatures are almost unobservable in the OS PSD. In addition, its CPLE-COH delineates meaningful correlation at these two bearing frequencies which further validates that the signal at $\mathrm{C}$ and I-2N are indeed RPM-related as a roller bearing signature supposes to be. The presence of these two bearing signals is by no means an indicator of a bearing defect 
since they can be found in most SSME test accelerometer data (with such OS+CPLE enhancement preformed) and have resulted in no bearing damage. However, their presence provides a valuable window (or media) to monitor bearing condition; i.e. develop Condition Indicators, by detecting any small frequency shift as bearing wear or defect develops. It should be pointed out that, without OS pre-processing, these bearing frequencies would have been virtually impossible to detect from the raw PSD due to RPM variation.

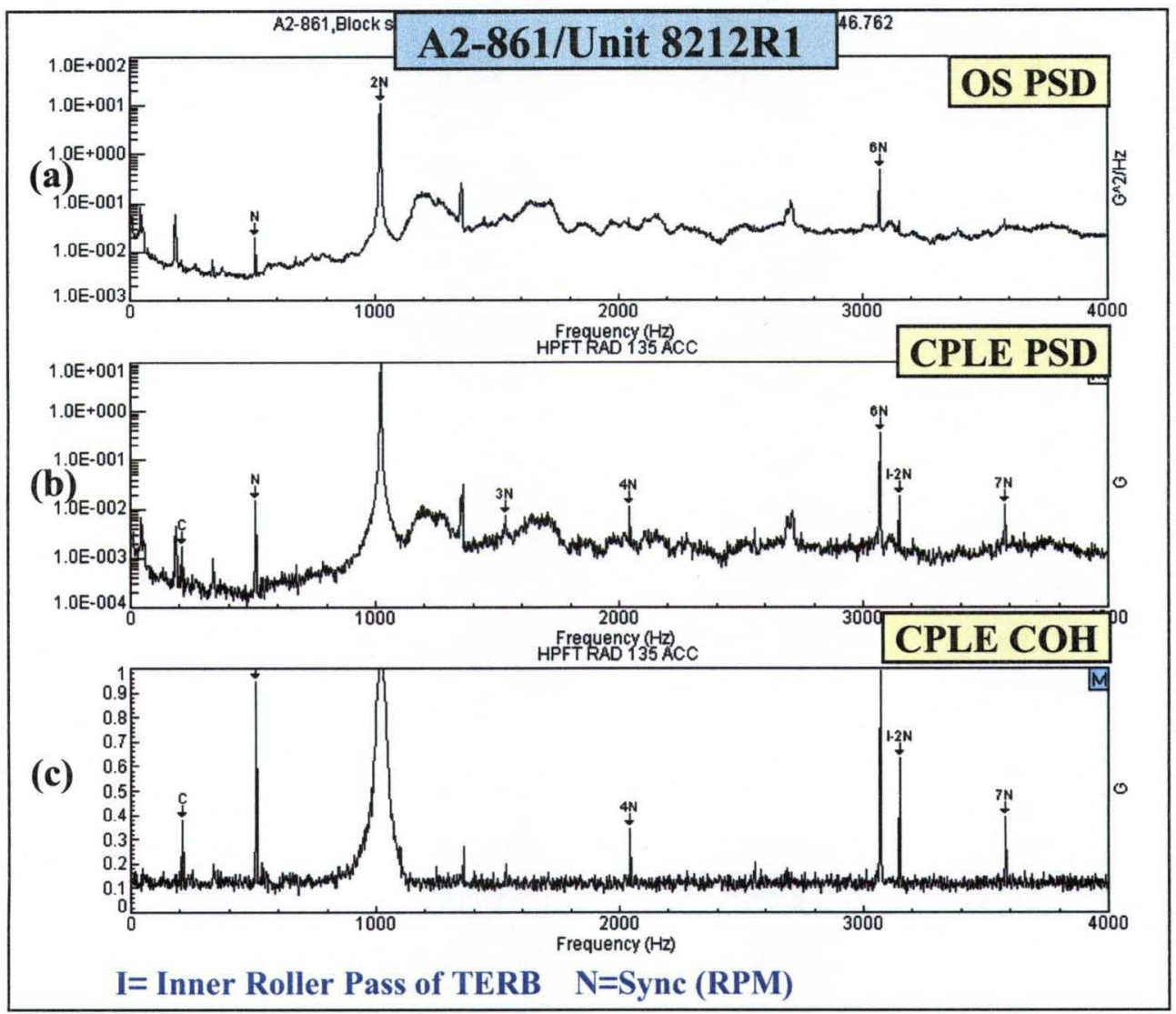

Figure 1 CPLE Spectral Analysis of a Turbine-End Accel measurement from SSME Ground test A2-861 (HPFTP Unit 8212R1).
(a) OS PSD
(b) CPLE-PSD
(c) CPLE-Coherence

Figure 2(a) shows the same CPLE-PSD from figure 1(b) with frequency zoomed around $\mathrm{I}-2 \mathrm{~N}$ spectral line $(\mathrm{OS}$ Frequency $=3150 \mathrm{~Hz})$. Since the frequency estimation from the FFT-based PSD is limited by its frequency resolution or bandwidth $\Delta$ (which is defined as Sampling Frequency/FFT Block Size or $2.5 \mathrm{~Hz}$ in this example), the actual frequency of I-2N could be anywhere within the frequency band of plus/minus half-bandwidth around the spectral line (i.e. $3150 \pm 0.5 \Delta$ ). Due to the additional signal power decomposition by CPLE analysis into a finer CPLE frequency band at each spectral line, it can further pinpoint the actual frequency within this band and provide more accurate frequency estimation as shown in figure 2(b). Figure 2(c) shows the CPLE Frequency spectrogram throughout the entire test period. Its X-axis represents the CPLE Frequency band around $\mathrm{I}-2 \mathrm{~N}$ spectral line (i.e. $3150 \pm 1.25 \mathrm{~Hz}$ ). Its $\mathrm{y}$-axis represents time. The corresponding RPM profile is shown on the left of the spectrogram. From such CPLE 
Frequency spectrogram, a more detailed frequency spectrum is obtained which shows the $\mathrm{I}-2 \mathrm{~N}$ bearing frequency remaining relatively constant throughout the test. This is an expected normal characteristic since all the roller bearing parameters (such as bearing diameter, pitch diameter and contact angle) remain unchanged. Ninety-eighty percent (98\%) of the 246 SSME tests analyzed for the baseline study show such characteristics at the I-2N CPLE Frequency. Also notice that when the RPM drops, around S+250 seconds, the bearing frequency tends to increase slightly. Such phenomenon is consistently observed in all other SSME tests which could be caused by a very small bearing geometry change due to load change.

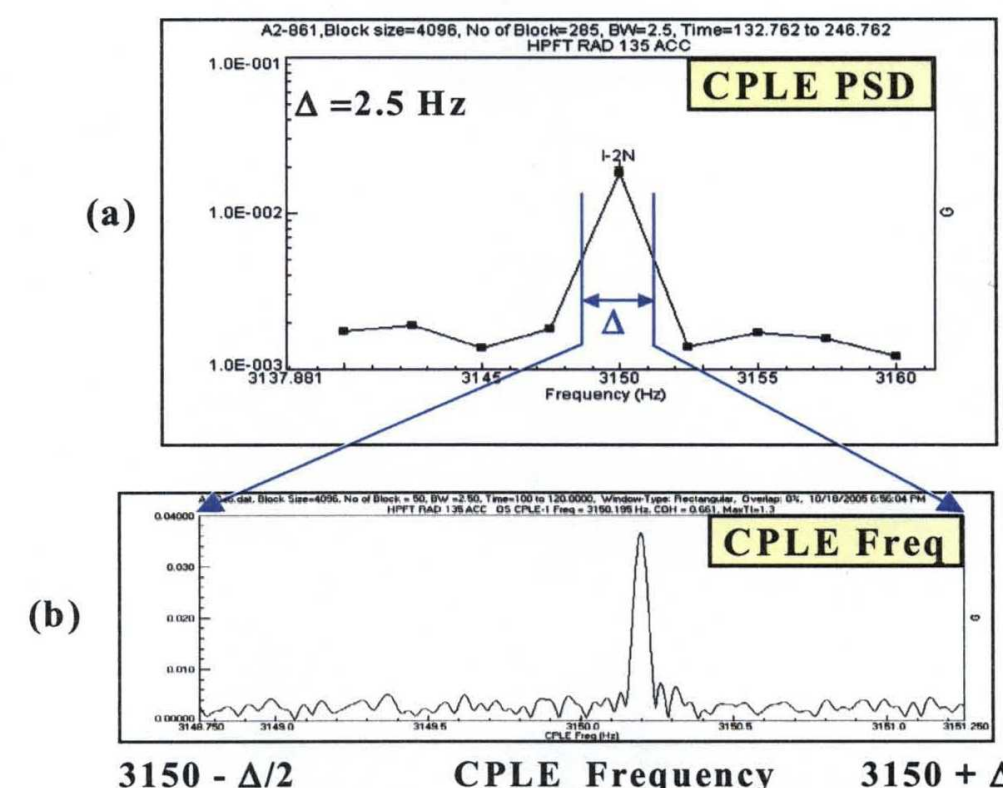

(c)

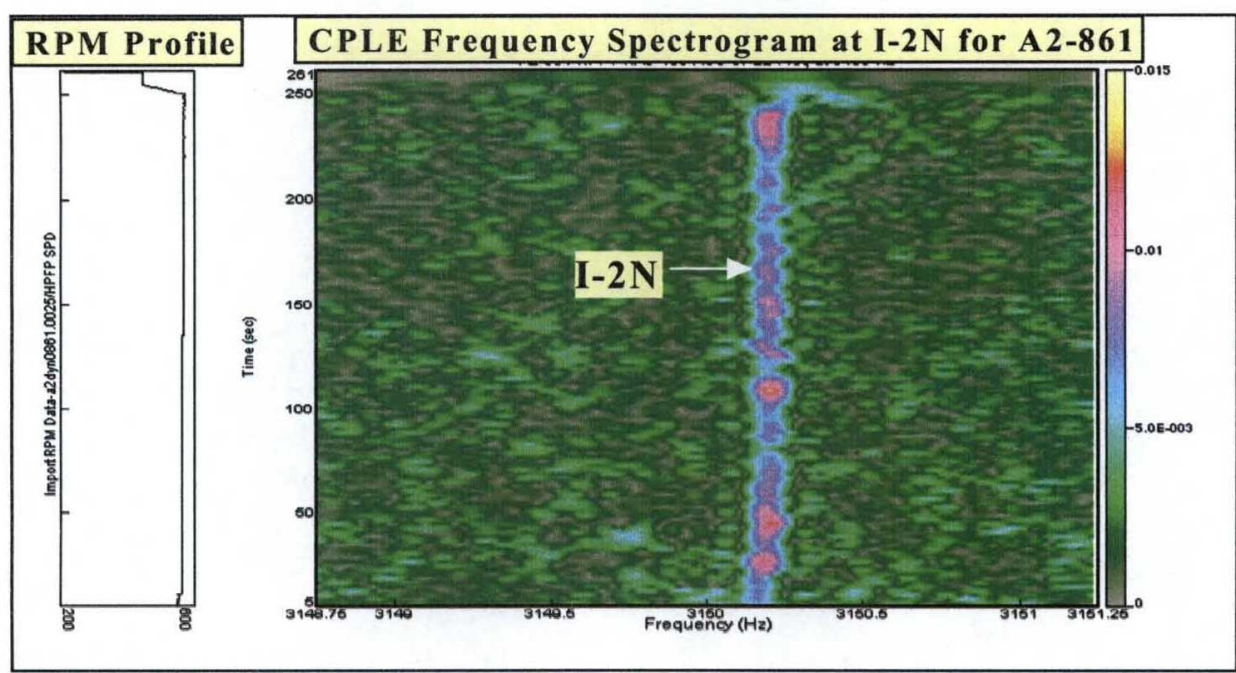

Figure 2 CPLE Frequency Analysis of a Turbine-End Accel measurement from SSME Ground test A2-861 (HPFTP Unit 8212R1).

(a) CPLE-PSD zoomed around I-2N spectral line at $3150 \mathrm{~Hz}$

(b) Signal power decomposes at I-2N into CPLE frequency band.

(c) CPLE Frequency spectrogram at I-2N throughout the entire test period. 
Figure 3 shows the CPLE-Frequency spectrogram at I-2N from another SSME test, 902846 with HPFTP Unit 8115R1. Unlike the previous result in Figure 2, an anomalous bearing frequency shift phenomenon is observed. The shift is very small (only about 0.5 $\mathrm{Hz}$ ), but it can be clearly observed by the CPLE Frequency. A TERB bearing Cage delamination was found in this turbopump bearing. During post-test inspection, small amounts of cage material were found to have peeled off the cage. Several other tests of this same unit also exhibited similar I-2N bearing frequency shifts. The relationship between such anomalous bearing frequency shifts and Cage delamination mechanism is not clearly understood yet. This example demonstrates the effectiveness of CPLE Frequency analysis in detecting those types of roller bearing defects which can cause small bearing characteristic frequency shifts. All HPFTP frequency shifts at I- $2 \mathrm{~N}$ that have been observed to date (on three HPFTP Units from $2 \%$ of the $246 \mathrm{SSME}$ tests), have resulted in shifts of the order of $\sim 0.5 \mathrm{~Hz}$.

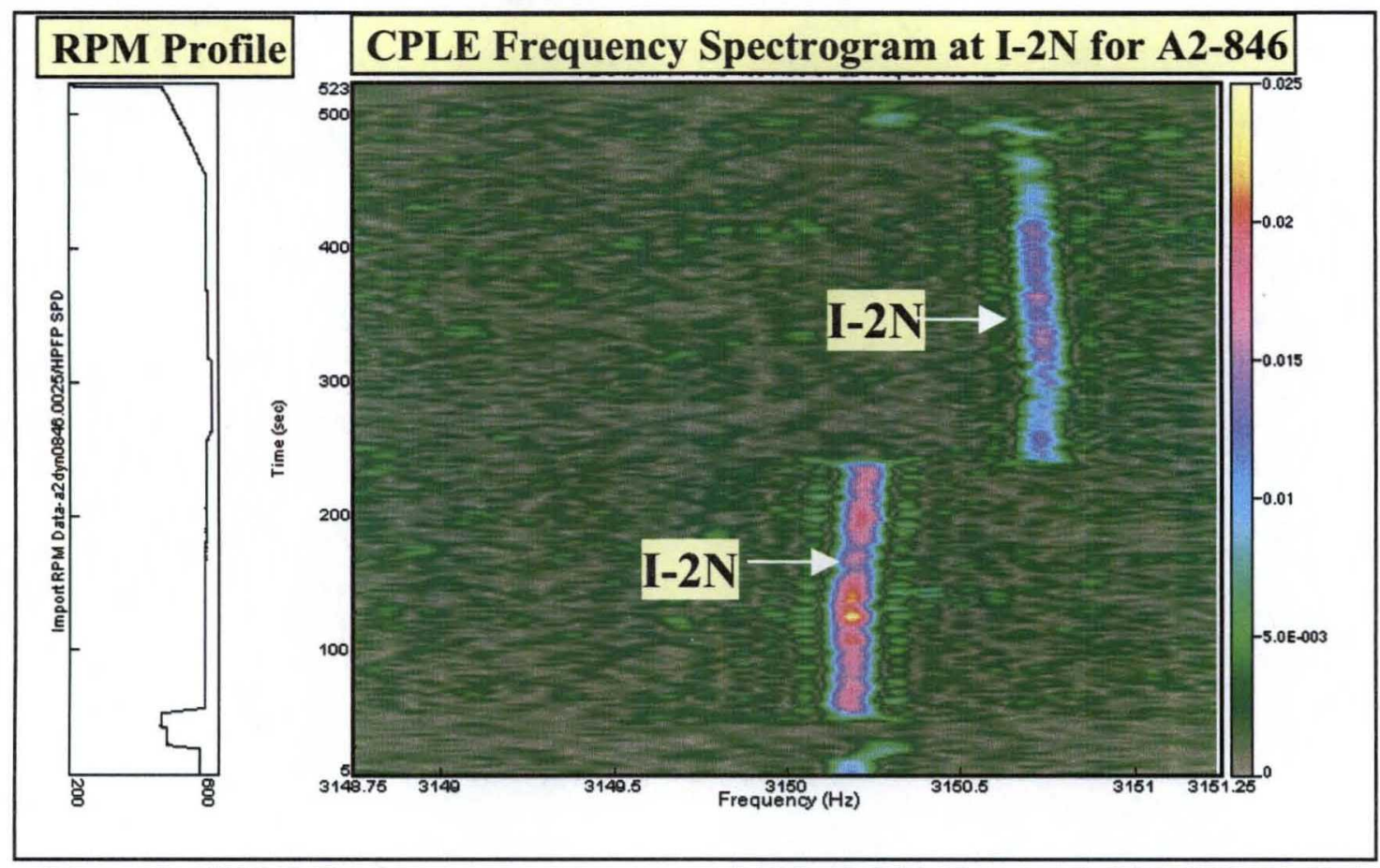

Figure 3 CPLE-Frequency spectrogram at I-2N of SSME test A2-846 HPFTP Unit $8115 \mathrm{R} 1$ with TERB bearing Cage Delamination found

Figure 4 shows similar analysis results for a recent Space Shuttle Flight, STS-115. The 4/Rev speed measurement for the HPFTP was not available during the flight. Therefore, the PKP technique was used to reconstruct a 1/Rev PKP signal directly from the instantaneous phase signal at $2 \mathrm{~N}$ ( $2^{\text {nd }}$ harmonic of Sync frequency) of an external accelerometer measurement. The resulting PKP signal was then used to perform OSpre-processing followed by CPLE Frequency analysis. Figure 4 shows the resulting CPLE-Frequency spectrogram at I-2N from STS-115 Main Engine 3 HPFTP 8118. Much like other nominal units, its I-2N frequency remains relatively constant throughout the flight which is an expected signature characteristic. This example demonstrates that even when a key phasor speed measurement is not available, it is still possible to perform CPLE Frequency analysis as an effective bearing health monitoring tool. 


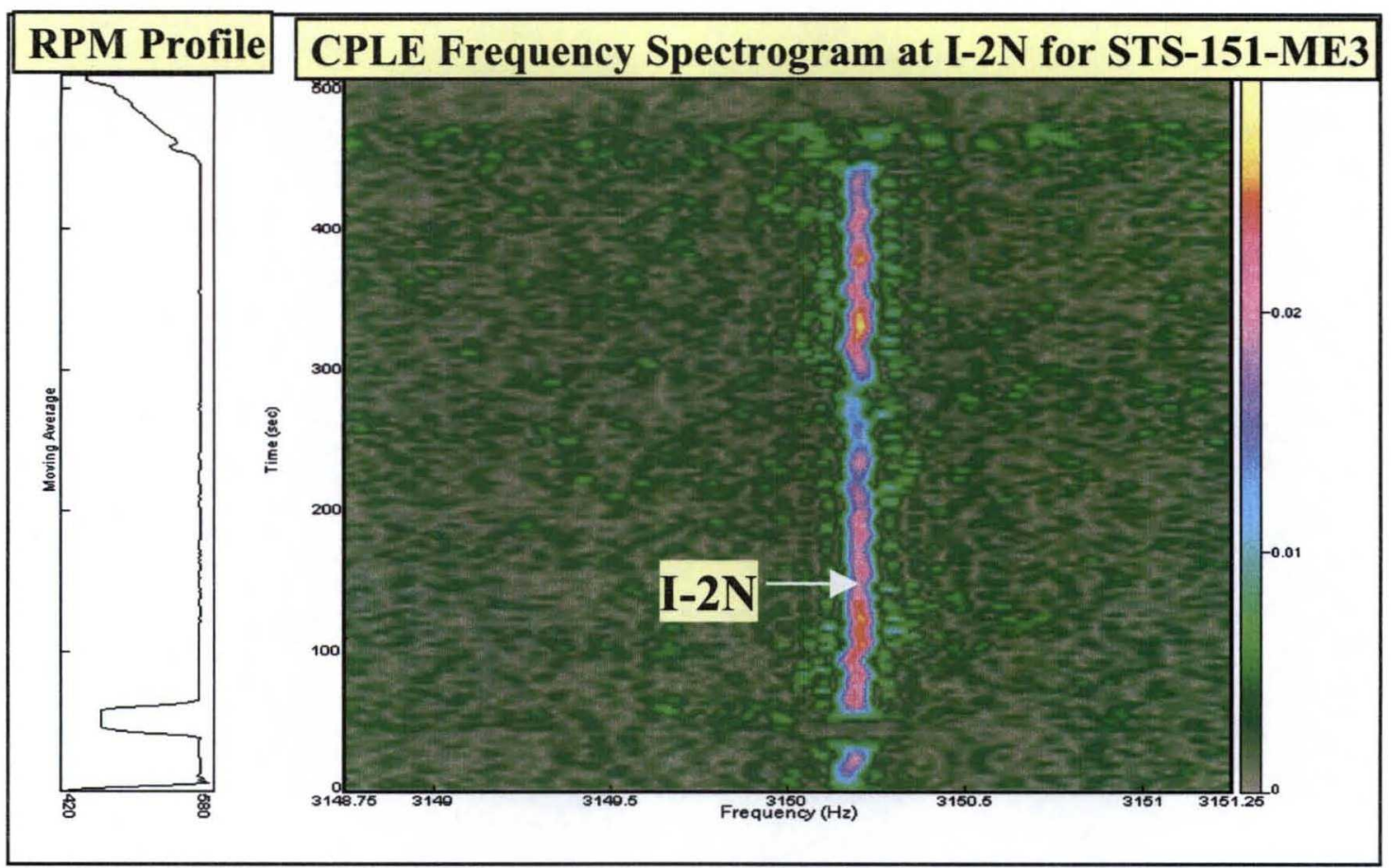

Figure 4 CPLE Frequency spectrogram at I-2N of STS-115 Main Engine 3 HPFTP using PKP to perform OS pre-processing.

References:

[1] J. Jong, W. Dorland, T. Fiorucci, T. Zoladz, T. Nesman "Coherent Phase Line Enhancer (CPLE) for Rotating Machinery Diagnostics" AIAA $37^{\text {th }}$ Joint Propulsion Conference, July 2001 (AIAA 2001-3761)

[2] T. Fiorucci, T. Reynolds "Application of the Space Shuttle Main Engine Real-Time Vibration Monitoring System for Advanced Engine Health Management" Joint $52^{\text {nd }}$ JANNAF Propulsion Meeting / $1^{\text {st }}$ Liquid Propulsion Subcommittee Meeting, May 2004. 


\title{
Roller Bearing Health Monitoring Using CPLE Frequency Analysis Method
}

\author{
Dr. Jen-Yi Jong ${ }^{1}$ and Jess H. Jones ${ }^{2}$ \\ AI Signal Research, Inc., Huntsville, AL 35805 \\ Tony Fiorucci ${ }^{3}$ \\ NASA/Marshall Space Flight Center, Marshall Space Flight Center, AL 35812
}

\begin{abstract}
This paper describes a unique vibration signature analysis technique - Coherence Phase Line Enhancer (CPLE) Frequency Analysis - for roller bearing health monitoring. Defects of roller bearing (e.g. wear, foreign debris, crack in bearing supporting structure, etc.) can cause small bearing characteristic frequency shifts due to minor changes in bearing geometry. Such frequency shifts are often too small to detect by the conventional Power Spectral Density (PSD) due to its frequency bandwidth limitation. This Coherent Phase Line Enhancer technology has been evolving over the last few years and has culminated in the introduction of a new and novel frequency spectrum which is fully described in this paper. This CPLE technology uses a "key phasor" or speed probe as a preprocessor for this analysis. With the aid of this key phasor, this CPLE technology can develop a twodimensional frequency spectrum that preserves both amplitude and phase that is not normally obtained using conventional frequency analysis. This two-dimensional frequency transformation results in several newly defined spectral functions; i. e. CPLE-PSD, CPLECoherence and the CPLE-Frequency. This paper uses this CPLE frequency analysis to detect subtle, low level bearing related signals in the High Pressure Fuel Pump (HPFP) of the Space Shuttle Main Engine (SSME). For many rotating machinery applications, a key phasor is an essential measurement that is used in the detection of bearing related signatures. There are times however, when a key phasor is not available; i. e. during flight of any of the SSME turbopumps or on the SSME High Pressure Oxygen Turbopump (HPOTP) where no speed probe is present. In this case, the CPLE analysis approach can still be achieved using a novel Pseudo Key Phasor (PKP) technique to reconstruct a 1/Rev PKP signal directly from external vibration measurements. This paper develops this Pseudo Key Phasor technique and applies it to the SSME vibration data.
\end{abstract}

\section{Introduction/Background}

$\mathrm{T}$ HE Space Transportation System (STS) has been operating since April 12, 1981. Since that time, the Space Shuttle Main Engine (SSME) has been the primary propulsion system used to propel the Space Shuttle Orbiter into orbit. The first test of the SSME was in 1975 and since that time over one million seconds of static (hot) firings and flight operations have been acuminated on the SSME. The one million seconds milestone was reached in January of 2004.

The Space Shuttle Orbiter utilizes three SSME's that provide approximately 1.2 million pounds of total thrust; i.e. $\sim 400,000$ pounds each. Each SSME is 14 feet long and weighs $~ 7,800$ pounds. The typical SSME burn time to orbit is approximately 520 seconds. The SSME uses Liquid Hydrogen (LH) and Liquid Oxygen (LOX) as its fuel and oxidizer, respectively. The major components for the SSME are shown in the expanded view in Figure 1. It has two low-pressure pumps, the Low Pressure Fuel Pump (LPFP) and the Low Pressure Oxidizer Pump (LPOP)

\footnotetext{
${ }^{1}$ Research Engineer, AI Signal Research, Inc, Huntsville, AL 35805, AIAA Member.

${ }^{2}$ Research Engineer, AI Signal Research, Inc, Huntsville, AL 35805, AIAA Member.

${ }^{3}$ Aerospace Engineer, NASA/MSFC/ER41, MSFC, AL 35812, Propulsion Systems Department.
} 
and two high-pressure pumps; the High Pressure Fuel Pump (HPFP) and the High Pressure Oxidizer Pump (HPOP). These pumps are mounted as shown in Figure 1. The high pressure pumps are attached to the "hot gas manifold" and form the main "powerhead" of the SSME. The actual hardware of the HPFP and LPFP mounted on the SSME is shown in Figure 2. The mated configuration for the SSME high pressure pumps on the "hot gas manifold" combined with the "main injector" and the "main combustion chamber" is as shown in Figure 3.
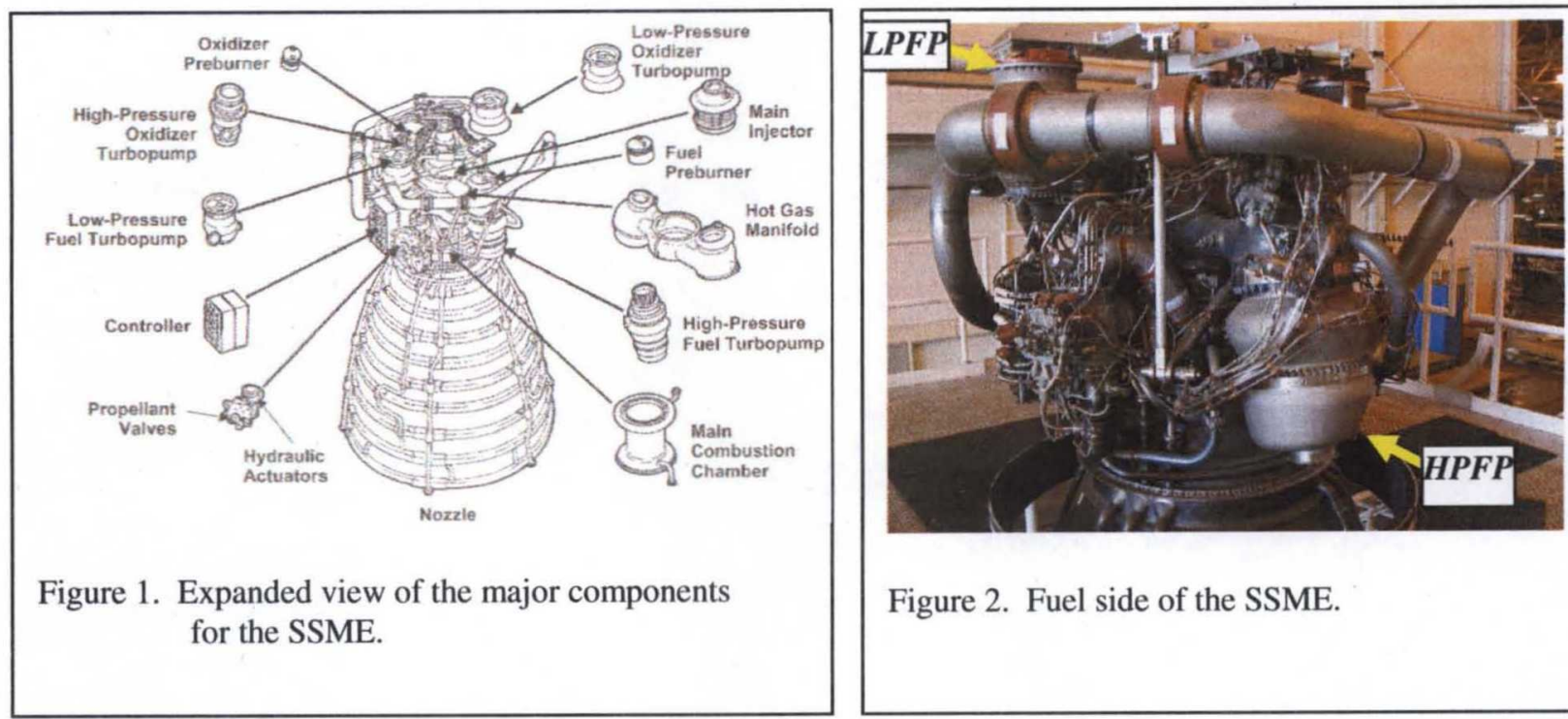

Figure 2. Fuel side of the SSME.

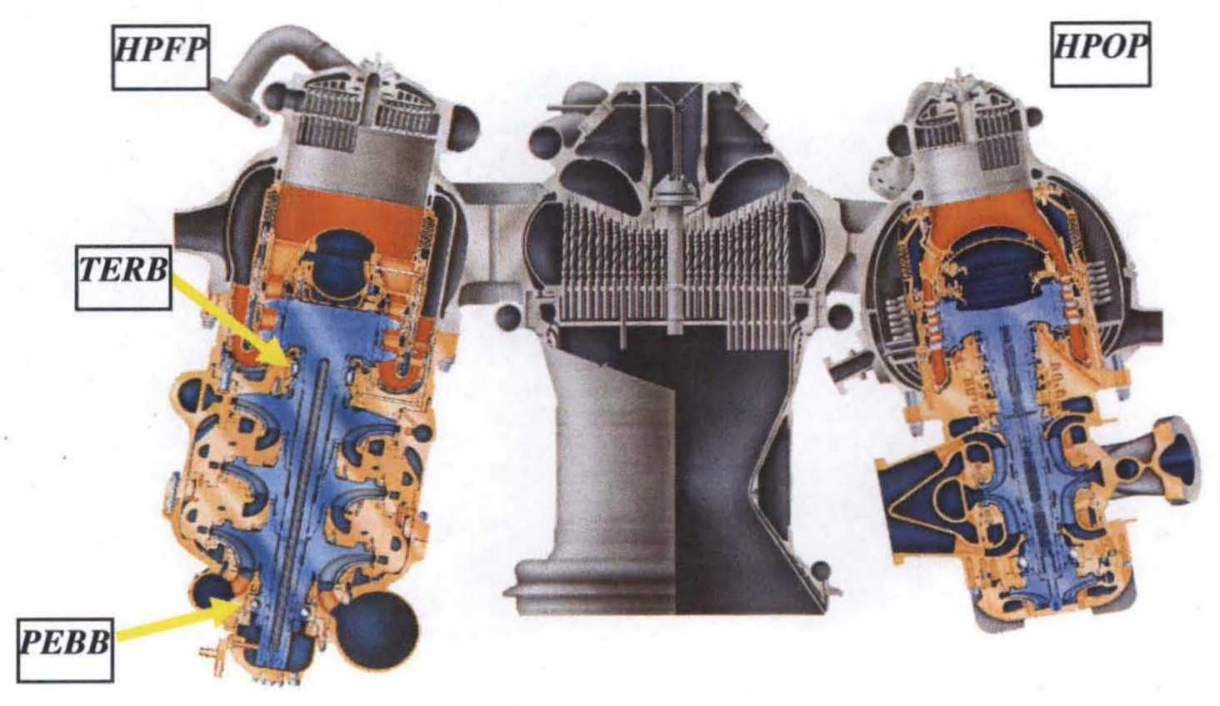

Figure 3. SSME high pressure pumps on the hot gas manifold with the main injector, main combustion chamber and the fuel/lox preburners. HPFP is on the left side and the HPOP is on the right 
During operation of the SSME, such as during a static (hot) firing or STS flight, a large number of vibration measurements are made in order to evaluate the operational/structural integrity of some of the major components of the engine. This evaluation must be made before the next test on these components is performed. As can be imagined, all components experience very severe, high level, vibration environments. There is high level random vibration caused by the combustion processes associated with the main combustion chamber/nozzle as well as other random excitations. In addition to this random excitation that is produced by the SSME, there are also "quasiperiodic" components generated by the rotating machinery; i.e. "turbopumps". Consequently, typical SSME vibration signatures/spectra contain a combination of random and periodic/quasi-periodic components.

One major focus of study of the SSME vibration signatures during these past years, has been the detection of low level periodic/quasi-periodic components in the presence of high level random background excitation. This paper presents yet another approach to this important problem. The approach described herein; i.e. the Coherence Phase Line Enhancer (CPLE) provides an effective means for detection of low level periodic/quasi-periodic components in the presence of a high level random environments. In particular, this technique is ideally suited to the detection of "roller bearing" signatures in the presence of high level background random environments. This CPLE approach has been evolving over the past several years as more experience has been acquired in its use/understanding and has culminated in the approach that is presented herein. The main focus of the dynamic analysis discussed here is with the detection of "turbine end roller bearing" (TERB) signatures on the High Pressure Fuel Pump (HPFP) of the SSME. The TERB of the HPFP can be seen in Figure 3. The HPFP generates 76,000 of shaft horsepower and its maximum rotational speed is $36,200 \mathrm{RPM}$.

The nominal rotational speed for the HPFP, at a Rated Power Level (RPL) of 104\%, is $\sim 563 \mathrm{~Hz}$; i.e. 33,780 RPM. The Turbine and Pump End bearing frequency ratios are shown in Table 1, where RE is the rolling element, ORP is the outer roller/ball pass and IRP is the inner roller/ball pass frequency ratios. The number of rolling elements for the turbine end bearing is 14 and for the pump end bearing is 11 .

\begin{tabular}{|c|c|c|c|c|}
\hline HPFP BEARNN FREQUENCY RATIOS & Cage & RE & ORP & IRP \\
\hline HPFTP/PEBB & $\mathbf{0 . 3 9 5 1 1}$ & $\mathbf{2 . 2 0 0 9}$ & $\mathbf{4 . 3 4 6 2}$ & $\mathbf{6 . 6 5 3 8}$ \\
\hline HPFTP/TERB & $\mathbf{0 . 4 1 7 7}$ & $\mathbf{2 . 9 5 5 2 2}$ & $\mathbf{5 . 8 4 7 7 5}$ & $\mathbf{8 . 1 5 2 2 5}$ \\
\hline \\
Table 1. SSME HPFP Bearing Frequency Ratios. \\
\hline
\end{tabular}

The material for the HPFP inner and outer races is Cronidur 30 and the rollers and balls are made of Silicon Nitride. The cage material for the turbine end bearing is made of Armalon and for the pump end material is Armalon w/Salox insert. 


\section{Development of CPLE Concept}

\section{A. Technical Analysis Background}

As indicated earlier, this CPLE Frequency Analysis ${ }^{1-2,4-18}$ approach is a culmination of several years of research effort. These past efforts include CPLE PSD for RPM-related signal enhancement, CPLE-Coherence for RPM correlation identification, CPLE-Frequency for high resolution frequency analysis, and the CPLE-TSA method which enhances conventional Time Synchronous Averaging (TSA) for gearbox signature analysis by incorporating an order validation capability using CPLE-Coherence to measure the quality of gear signatures and the associated Condition Indicators (CIs). When a speed measurement is not available, the PKP technique provides an alternative to enable the CPLE analysis by constructing a 1/rev PKP reference signal directly from a vibration measurement.

\section{B. CPLE Frequency Analysis Development}

During steady state operation of rotating machinery, the shaft rotational speed (RPM) tends to momentarily speed up or slow down due to dynamic load variation. As a result, the vibration signal associated with all the speedrelated components are quasi-periodic instead of periodic. Therefore, the CPLE technique first utilizes the Order Synchronized (OS) analysis method as a pre-processor to transform all speed-related quasi-periodic signal components into periodic components ${ }^{17,18}$. The OS method utilizes a $1 / \mathrm{rev}$ (or multiple/rev) pulse-tachometer-type signal (key phasor) to measure the instantaneous time per shaft revolution (i.e. time between pulses). Using this signal history, the original digital data stream that was sampled with uniform time intervals (i.e., time based sampling) is then resampled with a fixed number of samples during each revolution (i.e., shaft-revolution-speed based sampling; or constant phase sampling). Within the new resampled OS signal, each of the speed-related components become periodic. Thus, CPLE spectral analysis concentrates on all the periodic signal components within the OS signal, which now represent the speed-related components in the original signal.

The conventional method for estimating the Power Spectral Density (PSD) function is based on ensemble averaging the Fast Fourier Transform (FFT) amplitudes (Bendat ${ }^{3}$ ). This process first divides the time history of a measurement signal, $x(t)$, into a number of contiguous blocks. Each block of time data is then transformed into the frequency domain using the FFT algorithm. One benefit of this process is a smoother noise floor. This step averages the FFT amplitude squared over many ensemble blocks. A limitation of the process for performing PSD estimations is that only amplitude information is retained. All phase information is discarded. In other words, a valuable portion of the signal data (i.e. the phase) is discarded and not assessed.

The new CPLE technique incorporates phase information into the PSD averaging process ${ }^{13}$. The CPLE Spectrum resulting from this process is summarized in Figure 4. It provides spectral-enhancement which is the capability to detect RPM-related signals that are buried in broadband noise. This method is based on a unique coherent phase relationship in the ensemble domain as associated with a periodic signal. After Order Synchronization has been performed, this process then subdivides a time history of a measurement signal, $x(t)$, into a number of contiguous blocks, denoted by $y(m, t)$, where $m$ represents ensemble block number. Each block is then transformed into the frequency domain using FFT (with FFT size $=\mathrm{N}$ ):

$$
\begin{aligned}
& \mathrm{Y}(\mathrm{m}, \omega)=\text { Fourier Transform of } \mathrm{y}(\mathrm{m}, \mathrm{t}) \\
&= \frac{1}{N} \sum_{t=0}^{N-1} y(m, t) W_{N}^{\omega t} \\
& \text { where } W_{N}=e^{-j(2 \pi / N)}
\end{aligned}
$$

The resulting FFT, denoted by $\mathrm{Y}(\mathrm{m}, \omega)$, forms a 2-dimensional complex signal. The conventional PSD, $\mathrm{S}(\omega)$, is defined as:

$S(\omega)=\frac{2}{\Delta} \frac{1}{M} \sum_{m=1}^{M}|Y(m, \omega)|^{2}$ 
It can be shown that: If the signal, $x(t)$, is periodic at frequency $\omega$, then a coherent phase relationship will exist among different FFT blocks. This means that the complex signal $\mathrm{Y}(\mathrm{m}, \omega)$ is periodic in the ensemble direction. On the other hand, if the signal, $x(t)$, is random at $\omega$, such a coherent phase relationship will not exist since the FFT phases between different blocks are independent. Consequently, some potentially useful phase data exists in the ensemble direction that has not been exploited by the conventional PSD method.

Instead of ensemble averaging, the new CPLE method takes advantage of the phase correlation by performing another FFT (with FFTsize=M) in the ensemble direction to transform the complex signal, $\mathrm{Y}(\mathrm{m}, \omega)$, from the ensemble domain into a new "pseudo" wave-number domain, $\kappa$, and forms a new function, $\mathrm{Y}(\kappa ; \omega)$ :

$$
\begin{aligned}
& \mathrm{Z}(\kappa, \omega)=|\mathrm{Z}(\kappa, \omega)| \mathrm{e}^{-\mathrm{j} \phi(\kappa, \omega)}=\text { Fourier Transform of } \\
& \mathrm{Y}(\mathrm{m}, \omega)=\frac{1}{M} \sum_{m=0}^{M-1} Y(m, \omega) W_{M}^{\kappa m}
\end{aligned}
$$

It can be shown that: If the signal, $x(t)$, is periodic at frequency $\omega$, then its energy distribution, $Z(\kappa, \omega)$, will concentrate around a dominant peak at wave number, $\kappa_{\max }$, as depicted in Figure 5(a). Alternatively, if the signal, $x(t)$, is random at $\omega$, then the energy of $Z(\kappa, \omega)$ will be uniformly distributed over $\kappa$, as shown in Figure 5(b).

(a) $\mathbf{Z}\left(\kappa, \omega_{1}\right)$ for periodic signal

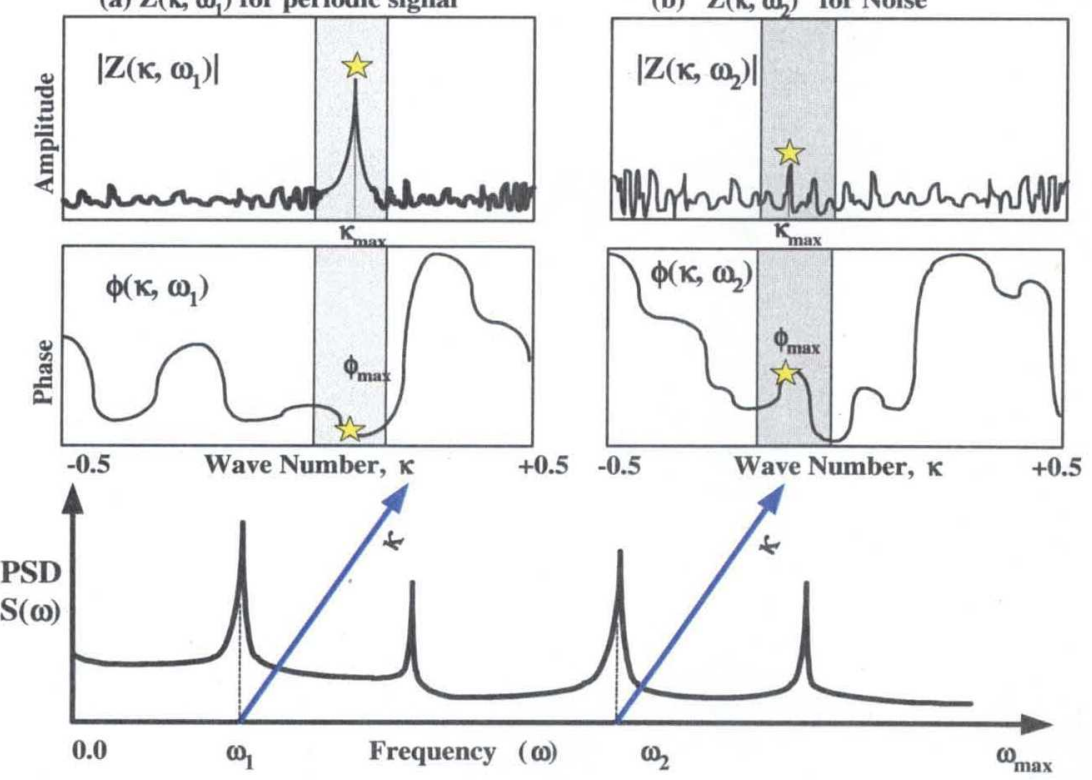

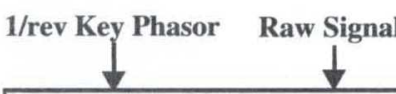

Order Synchronized (OS)

\begin{tabular}{|c|c|}
\hline \multirow{2}{*}{\multicolumn{2}{|c|}{\begin{tabular}{|c|} 
Block Segmentation within \\
stationary time frame: \\
$\mathrm{y}(\mathrm{t}, \mathrm{m}) \mathrm{t}=1, \mathrm{~N} ; \mathrm{m}=1, \mathrm{M}$ \\
\end{tabular}}} \\
\hline & \\
\hline & 2-D Signal( \\
\hline $\begin{array}{r}\text { 2-Dimensio } \\
\mathbf{Z}(\omega, \boldsymbol{\kappa})=\mathbf{F F T} \\
=\mid \mathbf{Z}(\omega,\end{array}$ & $\begin{array}{l}\text { al FFT: } \\
y(t, m)\} \\
\mid \exp \{\mathbf{j} \phi(\omega\end{array}$ \\
\hline & $2-1$ \\
\hline $\begin{array}{r}\text { Power Decor } \\
\text { Extract power } \\
\kappa_{\operatorname{Max}}=\operatorname{Max} P e \\
\text { in } \mathrm{Kdo}\end{array}$ & $\begin{array}{l}\text { nposition: } \\
\text { at } \kappa_{\operatorname{Max}} \\
\text { ak of }|Z(\omega, \kappa)| \\
\text { main }\end{array}$ \\
\hline & \\
\hline$S(\omega)=\sum_{\text {Entire }}$ & $\omega, \kappa) \mid 2$ \\
\hline$S_{C P L E}(\omega)=$ & $|Z(\omega, \kappa)|^{2}$ \\
\hline$S_{R E S}(\omega)=$ & $\sum_{\text {uding }}|Z(\omega, \kappa)|^{2}$ \\
\hline $\begin{array}{l}C_{C P L E}(\omega)= \\
\phi_{C P L E}(\omega)=\phi\end{array}$ & ${ }_{L E}(\omega)$ \\
\hline
\end{tabular}

Pre- processing w.r.t key-phasor

Figure 4. CPLE processing diagram

Figure 5. CPLE decomposition of $|\mathrm{Z}(\kappa, \omega)|$ in the Wave-Number ( $\kappa$ ) domain. 


\section{CPLE Spectrum}

Based on the previous observation, a new CPLE Spectrum, $S_{C P L E}(\omega)$, can be defined as:

$$
\text { CPLE Spectrum }=S_{C P L E}(\omega)=\frac{2}{\Delta} \sum_{\kappa=\kappa_{M a x}-N W}^{\kappa_{M a x}+N W}|Z(\kappa ; \omega)|^{2}
$$

Where: $\quad \kappa_{\mathrm{Max}}=$ wave number of the maximum peak of $|\mathrm{Z}(\kappa, \omega)|^{2}$ at $\omega$

$$
\begin{aligned}
\mathrm{NW}= & \text { CPLE wave number window width (= number of 1-sided wave-number bins around } \\
& \text { Kmax, the maximum peak). }
\end{aligned}
$$

The CPLE Spectrum basically represents the energy of $Y(\kappa, \omega)$ within a narrow CPLE wave number window around its maximum peak at $\kappa_{\max }$. Using the Parseval Relation, the following relationship can be established:

$$
\frac{1}{M} \sum_{m=0}^{M-1}|Y(m ; \omega)|^{2}=\sum_{\kappa=0}^{M-1}|Z(\kappa ; \omega)|^{2}
$$

This relationship states that the total energy of $\mathrm{Y}(\mathrm{m}, \omega)$ in the ensemble domain is equal to the total energy of $Z(\kappa, \omega)$ in the wave number domain. Therefore, as a special case, if the CPLE wave number window is fully open to cover the entire wave number ( -0.5 to 0.5$)$, then its CPLE spectra becomes identical to the conventional PSD. From these observations, it becomes clear that:

If the signal, $x(t)$, is periodic at frequency $\omega$, the CPLE wave number window captures a majority of energy of $Z(\kappa, \omega)$, because the energy distribution of $Z(\kappa, \omega)$ concentrates around a dominant peak at $\kappa_{\text {max }}$. As a result, the CPLE Spectrum approaches its conventional PSD amplitude. On the other hand, if the signal, $x(t)$, is random at $\omega$, the CPLE wave number window only captures a small portion of the total energy of $Z(\kappa, \omega)$ because the energy of $Z(\kappa, \omega)$ is uniformly distributed over $\kappa$. Consequently, the CPLE Spectrum will be much smaller than its conventional PSD.

This unique difference between a periodic signal and random noise in the wave number domain permits a noise reduction to be achieved by the CPLE spectra. The degree of noise reduction is calculated with the following equation.

$$
\text { Noise Reduction Level }=10 * \log _{10}\left\{\frac{2 * N W+1}{M}\right\} \mathrm{dB}
$$

This reduction level is simply the ratio of the width of CPLE wave number window to the entire wave number.

\section{CPLE Coherence}

As a result of the CPLE Spectrum, a new coherence function, called CPLE Coherence, can be developed. CPLE Coherence, a normalized CPLE Spectrum as defined below, provides a quantitative measure of the degree of discreteness of a spectral component.

$$
\text { CPLE Coherence }=C_{C P L E}(\omega)=\frac{\sum_{\kappa=\kappa_{\text {Max }}-N W}^{\kappa_{\text {Max }}+N W}\left|Z_{k}(\kappa, \omega)\right|^{2}}{\sum_{\kappa=-0.5}^{+0.5}\left|Z_{k}(\kappa, \omega)\right|^{2}}=\frac{C P L E \text { Spectra }}{\text { Conventional PSD }}
$$


The CPLE coherence represents the ratio of energy within the CPLE wave number window around its maximum peak at $\kappa_{\max }$ to its total energy. In other words, the CPLE coherence is simply the ratio between the CPLE spectra and the corresponding ordinary PSD. For a periodic signal, its energy $Z(\kappa, \omega)$, will concentrate around a dominant peak at $\kappa_{\max }$. Therefore, the CPLE Coherence will be nearly $100 \%$. In contrast, the energy $Z(\kappa, \omega)$, for broadband noise or a quasi-periodic signal, will be uniformly distributed over $\kappa$. Therefore, this CPLE Coherence is much smaller than unity. Thus, CPLE Coherence quantitatively measures the degree of discreteness of a signal frequency component. For machinery diagnosis, once the signal is speed normalized with respect to RPM using OS, CPLE Coherence represents a Speed-Coherence that quantitatively measures the degree of correlation of any spectral component with respect to (w.r.t.) speed.

\section{E. CPLE Frequency and CPLE Phase Spectrum}

The CPLE Spectrum and CPLE Coherence utilize the amplitude values of $Y(\kappa, \omega)$ (i.e. $|Y(\kappa, \omega)|)$. However, the phase of $Y(\kappa, \omega), \phi(\kappa, \omega)$, also represents useful values. For a speed-related signal component (e.g. the synchronous frequency super-harmonics, and sub-harmonics, gear-mesh. super- and sub- harmonic modulation, sidebands, etc), phase can be accurately calculated from $\phi(\kappa, \omega)$. This is due to another property of CPLE Spectrum that provides more accurate frequency estimation than a conventional PSD. The frequency resolution of a conventional PSD is limited by its spectral bandwidth, $\Delta$, defined as the sampling frequency divided by the FFT size:

$$
\Delta=\frac{F_{s}}{N}
$$

If a spectral peak in a conventional PSD is found at a particular frequency bin, $\mathrm{f}_{\text {bin }}$, then its actual frequency can be anywhere within $\pm 50 \% \Delta$ around that peak frequency $f_{\text {bin }}$. However, the CPLE spectrum further pinpoints the actual frequency within this $\pm 50 \% \Delta$ range. It can be shown that this location of the maximum peak at wave number domain, $\kappa_{\mathrm{max}}$, is closely related to the actual frequency in such a way that the actual frequency is simply equal to the CPLE frequency, $\mathrm{f}_{\mathrm{CPLE}}$, which is defined as follows:

$$
\text { CPLE Frequency }=\mathrm{f}_{\mathrm{CPLE}}=\mathrm{f}_{\text {bin }}+\kappa_{\max } * \Delta
$$

Consequently, the effective frequency resolution of a CPLE Spectrum is:

$$
\Delta_{C P L E}=\frac{\Delta}{M}
$$

The phase estimation from the FFT is also limited by its spectral bandwidth, $\Delta$. The FFT phase at a particular frequency bin, $\mathrm{f}_{\mathrm{bin}}$, is related to, but not equal to, its actual phase unless its actual frequency happens to coincide with the frequency bin. The CPLE Frequency, $\mathrm{f}_{\mathrm{CPLE}}$, accurately estimates the actual frequency within this $\pm 50 \% \Delta$ range. Therefore, the CPLE Spectrum further pinpoints the actual phase value. It can be shown that phase of $\mathrm{Y}(\kappa, \omega), \phi(\kappa, \omega)$, at the location of the maximum peak at wave number domain, $\kappa_{\mathrm{max}}$, provides an accurate measure of the actual phase as:

$$
\text { CPLE Phase Spectrum }=\phi_{C P L E}(\omega)=\phi\left(\kappa_{\max }, \omega\right)-\kappa_{\max } * \pi
$$

The CPLE Phase Spectral values represent the initial phase of speed-related periodic components.

\section{F. Theoretical Development of Pseudo Key Phasor (PKP) Method.}

As seen from the development of the CPLE Frequency Analysis approach described above, the initial input to this procedure requires the use of a "key phasor". In many applications however there is no key phasor (or speed probe) available. This is often due to safety considerations or to the inability to implement a key phasor as part of the rotating machinery. In these situations, the development of a Pseudo Key Phasor (PKP) is required. 
There are two major requirements for PKP application: (1) Stationary case during steady RPM (e.g. cruise mode, constant power/speed levels) and (2) Non-Stationary case during speed-up/coast-down (e.g. power/speed level change).

\section{PKP for Stationary Case}

A rotor system, as shown in Figure 6, generates excitation forces e $(\mathrm{t})$ at sync harmonic frequencies such as blade passage (BP) pressure waves or gear meshing (GM) force. The excitation forces in the rotational degree of freedom (DOF) vibrates the surrounding structure and generates vibration response $\mathrm{x}(\mathrm{t})$ in the transversal $\mathrm{DOF}$ at the $\mathrm{BP}$ or GM frequency. During stationary RPM operation, the excitation force is usually quasi-periodic (rather than periodic) due to the load variation on the rotor shaft. Therefore, such rotational excitation force and transversal vibration response can be modeled as a narrow band random signal ${ }^{15,16}$ :

$$
\begin{aligned}
& \mathrm{e}(\mathrm{t})=\mathrm{E}(\mathrm{t}) \cos [\mathrm{K} * \mathrm{~N} * \mathrm{t}+\alpha(\mathrm{t})] \\
& \mathrm{x}(\mathrm{t})=\mathrm{X}(\mathrm{t}) \cos [\mathrm{K} * \mathrm{~N} * \mathrm{t}+\beta(\mathrm{t})]
\end{aligned}
$$

Where: $\quad E(t), X(t)=$ Instantaneous amplitude of $e(t)$ and $x(t)$

$\alpha(t), \beta(t)=$ Instantaneous phase of $e(t)$ and $x(t)$

$\mathrm{N}=$ Center frequency of constant RPM (Sync)

$\mathrm{K}=$ Harmonic number of BP or GM frequency

The phase of the excitation force, $\alpha(t)$, is directly synchronized with the shaft rotational process in the rotational DOF. The phase of vibration response, $\beta(t)$, of the surrounding structure in the transversal DOF is controlled by the input excitation force and the system's transfer function. A tachometer key phasor measures the phase of the input excitation motion in the rotational DOF, while a vibration sensor measures system output response in the transversal DOF. For a linear system with transfer function of:

$$
H(f)=|H(f)| e^{j \phi(f)}
$$

the input excitation and output response are coherently phase-related to each other, governed by the phase of the transfer function $\phi(f)$ as:

$$
\beta(t)-\alpha(t)=\phi(f)
$$

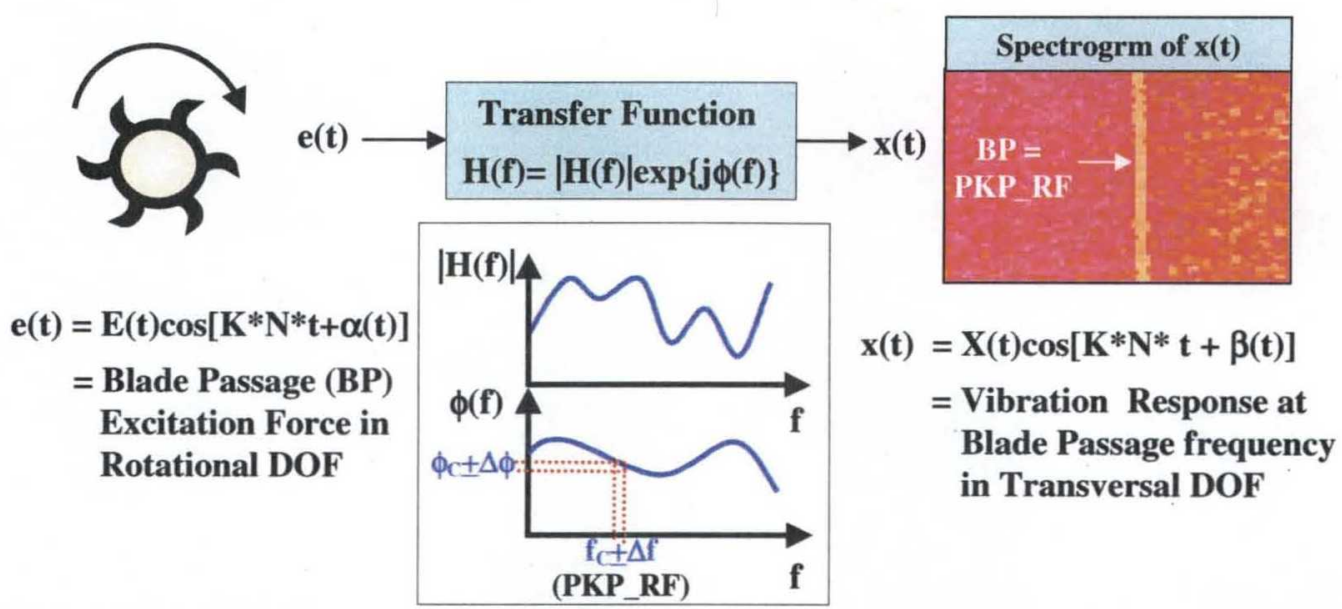

Figure 6. -Relationship between excitation force $e(t)$ in rotational DOF and vibration response $x(t)$ in transversal DOF during stationary RPM.

During stationary operation when RPM remains relatively constant, its Sync harmonics such as BP or GM frequency (which is referred to as PKP Reference Frequency, PKP_RF) will also be relatively constant with small frequency variation $\Delta \mathrm{f}$ : 


$$
\text { PKP_RF }=\mathrm{f}_{\mathrm{c}} \pm \Delta \mathrm{f}
$$

If the transfer function phase around PKP_RF remains relatively constant with a small phase variation $\Delta \phi$ :

$$
\phi(\text { PKP_RF })=\phi_{\mathrm{c}} \pm \Delta \phi
$$

then the phase difference between input and output signal will also be a relatively constant phase:

$$
\beta(\mathrm{t})-\alpha(\mathrm{t})=\phi(\text { PKP_RF })=\phi_{\mathrm{c}} \pm \Delta \phi
$$

This way, a constant time-shifted version of $\alpha(t)$ in the rotational DOF can be recovered from $\beta(t)$ in the transversal vibration measurement to generated valid PKP signal.

Therefore, the basic requirement for stationary PKP application is that the PKP_RF's variation, $\Delta \mathrm{f}$, during PKP processing time frame (i.e. monitoring cycle) should remain within a small acceptable range so that its transfer function phase remains constant within PKP-RF variation (i.e. within $f_{c} \pm \Delta f$ ). During on-line or off-line monitoring, PKP will perform RPM/Performance data gating to search for a qualified stationary time frame with constant PKP-RF (i.e. $\Delta \mathrm{f}<$ acceptable limits) to ensure the requirement is met for stationary PKP application.

The PKP technique utilizes a phase/time relationship to convert the instantaneous phase of a vibration measurement at PKP_RF into a new 1/Rev pulse train PKP signal whose $\mathrm{N}$-th order harmonic possesses the same phase characteristic as the original vibration measurement at PKP_RF (e.g. BP or GM frequency). Figure 7 shows the signal processing diagram for Stationary PKP Generation. To initiate PKP Generation during each monitoring cycle, it first performs RPM gating or other performance data gating in order to track the desired PKP-RF component and search for qualified PKP processing time frame (i.e. monitoring cycle). The PKP-RF must correspond to a known RPM-related vibration component such as a gear-mesh or blade-passage component or any sync harmonic. Next, complex demodulation is performed to estimate instantaneous phase at the PKP-RF frequency. Based on a phase/time conversion relationship, the instantaneous phase $\phi(t)$ is then input to a digital oscillator to generate a constant-amplitude sinusoidal signal at PKP-RF. This sinusoidal signal has the same phase characteristic as manifested in the vibration measurement at PKP-RF. Subsequent waveform thresholding and decimation is performed to generate a $1 /$ Rev pulse train as the output PKP reference signal.

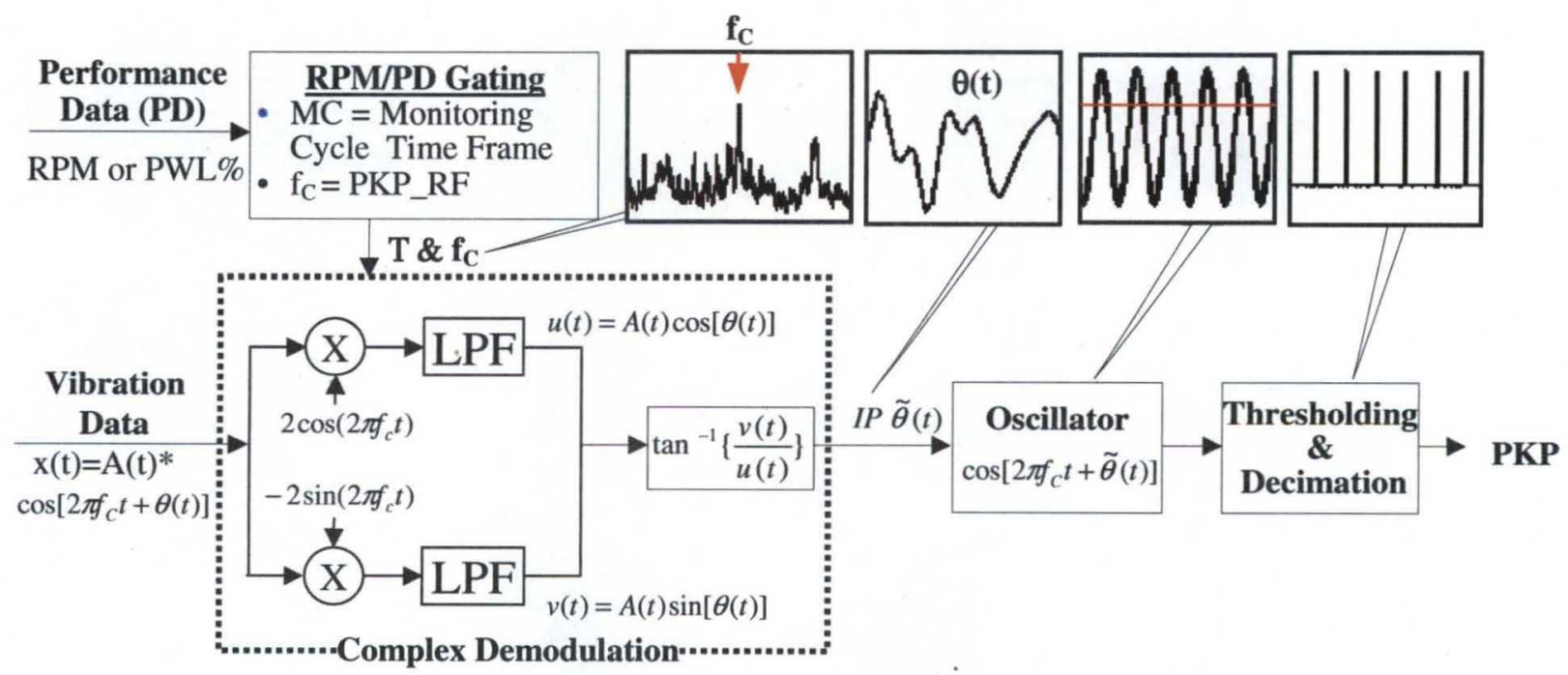

Figure 7. Stationary PKP generation flow diagram.

For a successful PKP generation, the resulting PKP signal will have a fundamental pulse train frequency at sync frequency $(\mathrm{N})$ with its instantaneous phase (IP) at $N$ being proportional to the IP at PKP_RF $=K * N$ in the original vibration measurement. In other words, PKP basically generates the K-th order sub-harmonic of PKP-RF (i.e. from $\mathrm{K} * \mathrm{~N}$ to $\mathrm{N}$ ) by maintaining its phase characteristic. 
After PKP is generated, the next step is to validate its credibility. Figure 8 shows the signal processing flow diagram for stationary PKP validation. A validation parameter called the Tracking Index (TI) is first computed to ensure it is within an acceptable level.

$$
T I=\text { Max }\left\{\frac{\text { Previous PKP Pulse Period }}{\text { Current PKP Pulse Period }} \%\right\} \text { of all PKP within monitoring cycle }
$$

Such TI validation ensures no abrupt change in key phase pulse periods from revolution to revolution. It can also eliminate any invalid PKP due to weak SNR at PKP-RF. During stationary operation, the TI should maintain around $100 \%$. If a missing pulse occurs in the PKP signal, its TI will jump to around $200 \%$. Therefore, as a rule of thumb, a TI threshold level setting of $160 \%$ should be adequate.

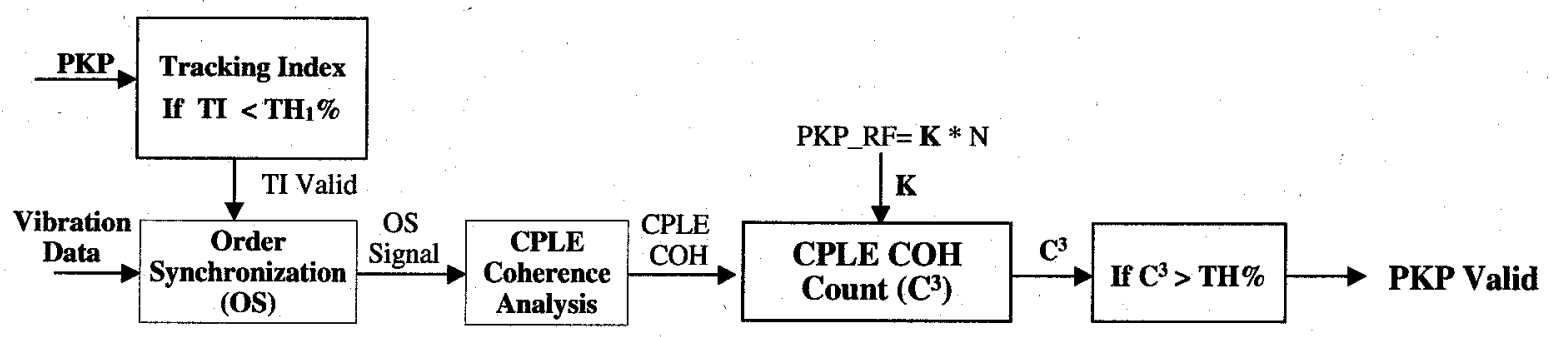

Figure 8. Stationary PKP validation flow diagram.

Once TI is validated, a second step for PKP validation will require the CPLE Spectral Analysis. The CPLECoherence function has a unique capability to measure the nonlinear correlation of a vibration component at any frequency with respect to RPM. The second validation is based on a discriminator whose phase variation (for a Valid PKP) at PKP frequency (PKP frequency =PKP_RF/K = K-th Sub-harmonic of PKP-RF) is expected to be identical to RPM's phase variation, resulting in all the harmonics of PKP being identical to true Sync harmonics. On the other hand for an erroneous PKP, its phase variation at PKP frequency will be independent of RPM and as a result, its PKP Harmonics will not be the same as true Sync harmonics. Therefore, this validation simply measures the CPLE-COH at all PKP harmonics to identify if they are true Sync harmonics or not. This is achieved by first performing Order Synchronization (OS) of the raw vibration measurement using the un-validated PKP as the reference signal. For a valid PKP signal, the resulting CPLE-Coherence after OS should display high RPM correlation at all sub-harmonics of PKP-RF while for an erroneous PKP, no such RPM correlation will be found. A validation parameter, called "CPLE Coherence Count $\left(\mathrm{C}^{3}\right)$," is computed from the CPLE-Coherence function.

$$
C^{3}=\left\{\frac{\# \text { of PKP Harmonics with CPLE }-\mathrm{COH}>\mathrm{TH}_{2}}{\text { Max Harmonic Number }}\right\} \%
$$

The $\mathrm{C}^{3}$ parameter represents the percentage of PKP harmonics being true Sync harmonics out of a pre-defined max harmonic\#. For a valid PKP, since all PKP harmonics should be identical to true Sync harmonic, therefore its CPLE-Coherence should shows strong correlation at many PKP harmonics. As a result, a high C3 will be obtained indicating valid PKP. Conversely, an erroneous PKP produces low CPLE-Coherence at each PKP harmonic and results in a very low $\mathrm{C}^{3}$ value. The PKP Validation also produces a unique sensor validation capability, because a failed sensor is highly unlikely to produce high CPLE-Coherence at any PKP-RF. During on-line monitoring, such $\mathrm{TI}$ and $\mathrm{C} 3$ provide effective parameters to validate the PKP before it is utilized for any subsequent analysis. Once a PKP signal is generated and validated, all vibration signature analysis that requires a 1/rev Key Phasor, such as the OS, TSA or CPLE, become applicable for fault detection and diagnostic evaluation.

Valid application of PKP imposes two major requirements. First, a strong RPM-related signal component must exist in the vibration measurement at PKP-RF to allow accurate phase estimation. This requirement is usually achievable for most machinery applications (e.g., SSME, aircraft gas turbine or power plant steam turbines), because it is quite common for an external vibration sensor to pickup strong blade-passage (BP) or gear mesh (GM) vibration responses. The second requirement is that the frequency variation of PKP-RF within the PKP generation time frame must be small enough so that the phase of its transfer function remains constant to ensure accurate recovery of a time-shifted input signal in the rotational DOF. 


\section{PKP for Non-Stationary Case}

As discussed earlier, the requirement for a valid PKP application during stationary RPM is that the transfer function phase around PKP-RF remains relatively constant. When this requirement is satisfied, a time-shifted version of instantaneous phase in the rotational DOF can be recovered from the instantaneous phase in the transversal DOF to generated PKP. This requirement is not difficult to meet unless the frequency variation of PKP$\mathrm{RF}$ becomes too large and becomes non-stationary (changing greatly). Since many anomalies/failure investigations of engine test/flight data often requires its vibration characteristics be examined during non-stationary operating conditions, it would be valuable to expand the applicability of PKP to the non-stationary case.

For non-stationary PKP application when RPM changes over a wider range, the rotational excitation and transversal response at PKP_RF become a narrow band signal with time-varying center frequency $\mathrm{N}(\mathrm{t})$, as:

$$
\begin{aligned}
& \mathrm{e}(\mathrm{t})=\mathrm{E}(\mathrm{t}) \cos \left[\mathrm{K}^{*} \mathrm{~N}(\mathrm{t}) * \mathrm{t}+\alpha(\mathrm{t})\right] \\
& \mathrm{x}(\mathrm{t})=\mathrm{X}(\mathrm{t}) \cos [\mathrm{K} * N(\mathrm{t}) * \mathrm{t}+\beta(\mathrm{t})] \\
& \text { Where: } \quad N(\mathrm{t})=\text { time-varying RPM frequency (Sync) }
\end{aligned}
$$

Figure 9 shows the flow diagram for non-stationary PKP generation. Similar to the stationary case, it utilizes a phase/time conversion to transform the instantaneous phase extracted from a vibration measurement at PKP_RF into a 1/Rev PKP signal. Since RPM frequency is no longer constant, a time-varying complex demodulation is required for instantaneous phase estimation. This requires the algorithm to first perform frequency tracking from the PSD of vibration data or from some other performance data (e.g. PWL\% or RPM Profile) to find PKP_RF. Since PKP_RF is a time-varying frequency, it is formulated as a piecewise linear varying frequency based on the block-to-block frequency tracking result. This piecewise linear varying frequency is then fed into the complex demodulator as its referenced signal to perform time-varying complex demodulation and instantaneous phase estimation at nonstationary PKP_RF.

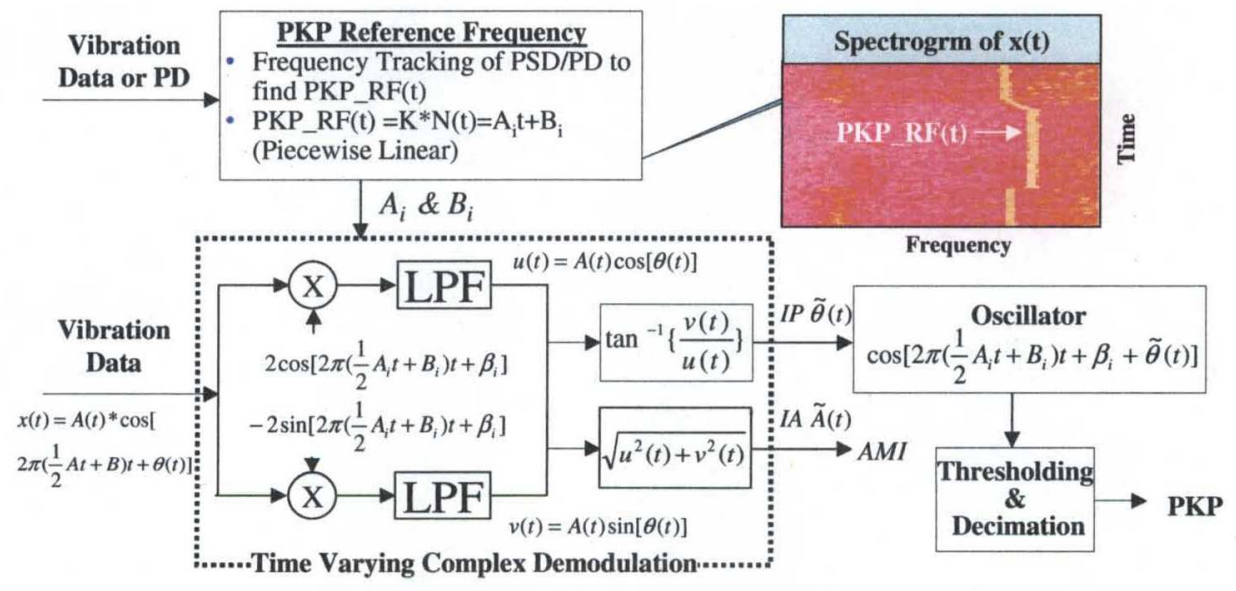

Figure 9. Non-stationary PKP generation flow diagram.

For a successful PKP generation, the instantaneous phase at the fundamental PKP frequency (PKP_RF/K) should be synchronized (proportional) with the instantaneous phase in the vibration measurement. The basic requirements for successful non-stationary PKP generation are that the vibration measurement must have a strong Signal-ToNoise Ratio at PKP-RF(t) to allow accurate instantaneous phase estimation. To ensure this, the Tracking Index (TI), along with another validation parameter called Amplitude Modulation Index (AMI), is computed for non-stationary PKP validation. The definition of TI is the same as the stationary case as defined in equation (17). The AMI is defined as:

$$
A M I=\frac{\text { Minimum of } X(t)}{\text { Average of } X(t)} \%
$$

Where: $X(t)=$ The instantaneous amplitude (i.e. Envelop signal) of vibration measurement $x(t)$ at PKP_RF 
The AMI is very similar to the modulation index for an Amplitude Modulation (AM) signal in communication theory ${ }^{19}$. If the signal at PKP_RF remains strong during the non-stationary time period, its AMI will maintain unity indicating a well-behaved waveform amplitude oscillating from cycle to cycle to allow accurate instantaneous phase estimation. If the SNR gets weaker, its AMI will reduce since its minimum envelope signal, $\mathrm{X}(\mathrm{t})$, will reduce. As the SNR drops to such a low level that it's envelop starts touching the ground (zero) level, ambiguity will be produced for instantaneous phase estimation that impacts the accuracy of the resulting PKP signal. In general, a threshold level of AMI from $10 \%$ to $25 \%$ range will be adequate (the higher threshold, the more reliable).

In addition to the two validation parameters, there are other important factors that will influence the accuracy of PKP for non-stationary case. As shown in Figure 10, as PKP_RF varies from $\mathrm{K}^{*} \mathrm{~N} 1$ to $\mathrm{K} * \mathrm{~N} 2$., the phase difference between the rotational DOF and transversal DOF will also change depending on the transfer function phase within the PKP_RF(t) variation:

$\beta(\mathrm{t})-\alpha(\mathrm{t})=\phi[$ PKP_RF(t)]

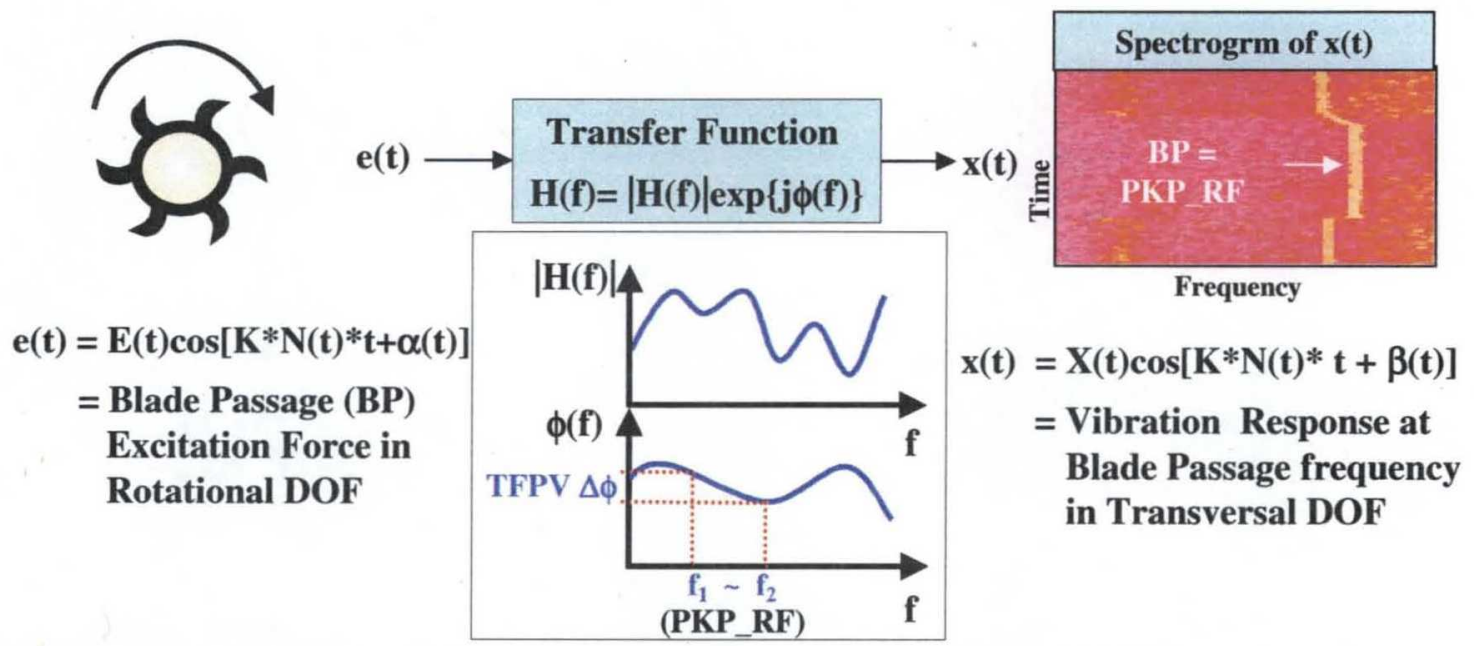

Figure 10. - Relationship between excitation force $e(t)$ in rotational DOF and vibration response $x(t)$ in transversal DOF during non-stationary RPM.

Any phase variation of its transfer function at PKP_RF will result in phase error in the resulting PKP. Therefore, it is critical to assess such PKP phase error for non-stationary application. To do so, let:

$\phi(f)=$ Transfer function phase between the rotational DOF and an Accel measurement in the transversal DOF used for PKP generation at PKP_RF=K*N

$\alpha(\mathrm{f})=$ phase of a True Key Phasor (TKP) signal (assume it's available)

$\beta(f)=$ phase of the Accel signal $[$ Note: $\beta(f)-\alpha(f)=\phi(f)]$

$\gamma(f)=$ phase of $1 / \operatorname{Rev}$ PKP signal

$\theta(\mathrm{f})=$ phase of the $\mathrm{K} / \mathrm{Rev}$ pulse train signal before it is decimated to generate $1 / \operatorname{Rev}$ PKP

Two parameters, TFPV and PKP_PE, are defined as:

PKP_PE (PKP Phase Error $)=[\gamma(\mathrm{N} 2)-\alpha(\mathrm{N} 2)]-[\gamma(\mathrm{N} 1)-\alpha(\mathrm{N} 1)]$

$=$ Variation of the phase difference between PKP and TKP at Sync frequency as it changes from N1 to N2 within the non-stationary time frame

TFPV (Transfer Function Phase Variation $)=\phi(K * N 2)-\phi(K * N 1)$ 
= Variation of transfer function phase between rotational DOF and transversal DOF at PKP_RF as it changes from $\mathrm{K}^{*} \mathrm{~N} 1$ to $\mathrm{K}^{*} \mathrm{~N} 2$ within the non-stationary time frame.

The basic requirement for non-stationary PKP application is that the PKP_PE must be within an acceptable range for the subsequent Sync Vector analysis using PKP. If the purpose is to search for a 180-degree phase shift phenomenon for resonance identification, then a PKP phase error of less than 10 degrees (5.5\%) should be considered acceptable.

A relationship between TFPV and PKP_PE is found that provides a useful way to estimate the PKP_PE for a particular pump, at particular measurement locations from its transfer function.

The PKP algorithm first generates a K/Rev pulse train signal from an Accel's PKP_RF $\left(=\mathrm{K}^{*} \mathrm{~N}\right)$, therefore:

$$
\beta\left(K^{*} N\right)=\theta\left(K^{*} N\right)
$$

Subsequently, the PKP algorithm then decimates the K/Rev pulse train signal to generate a 1/Rev PKP signal. As a result, the fundamental frequency of PKP becomes the K-th order sub-harmonic of K/Rev signal. Based on the theory of nonlinear coherence analysis ${ }^{10,11,20}$, a coherent phase relationship exists between a fundamental component and its $\mathrm{K}$-th order harmonic. Therefore, for TKP signal:

$$
\mathrm{K}^{*} \alpha(\mathrm{N})-\alpha\left(\mathrm{K}^{*} \mathrm{~N}\right)=\mathrm{C} 2 \text { (constant phase) }
$$

Similar phase relationship should also exist between the fundamental component of 1/Rev PKP signal and the K-th order harmonic of the $\mathrm{K} / \mathrm{Rev}$ signal:

$$
\mathrm{K}^{*} \gamma(\mathrm{N})-\theta(\mathrm{K} * \mathrm{~N})=\mathrm{C} 1 \text { (constant phase) }
$$

If equation (23) is multiplied by a constant $\mathrm{K}$ :

$$
\begin{array}{rlrl}
\mathrm{K}^{*} \mathrm{PKP} \_\mathrm{PE}= & \mathrm{K}^{*}\{[\gamma(\mathrm{N} 2)-\alpha(\mathrm{N} 2)]-[\gamma(\mathrm{N} 1)-\alpha(\mathrm{N} 1)]\} & \\
= & \left\{\left[\theta\left(\mathrm{K}^{*} \mathrm{~N} 2\right)+\mathrm{C} 1\right]-\left[\alpha\left(\mathrm{K}^{*} \mathrm{~N} 2\right)+\mathrm{C} 2\right]\right\}- & & \\
& \left\{\left[\theta\left(\mathrm{K}^{*} \mathrm{~N} 1\right)+\mathrm{C} 1\right]-\left[\alpha\left(\mathrm{K}^{*} \mathrm{~N} 1\right)+\mathrm{C} 2\right]\right\} & & \text { (based on euation 26 \& 27) } \\
= & {\left[\theta\left(\mathrm{K}^{*} \mathrm{~N} 2\right)-\alpha\left(\mathrm{K}^{*} \mathrm{~N} 2\right)\right]-\left[\theta\left(\mathrm{K}^{*} \mathrm{~N} 1\right)-\alpha\left(\mathrm{K}^{*} \mathrm{~N} 1\right)\right]} & & \\
= & {\left[\beta\left(\mathrm{K}^{*} \mathrm{~N} 2\right)-\alpha\left(\mathrm{K}^{*} \mathrm{~N} 2\right)\right]-\left[\beta\left(\mathrm{K}^{*} \mathrm{~N} 1\right)-\alpha\left(\mathrm{K}^{*} \mathrm{~N} 1\right)\right]} & & \text { (based on equation 25) } \\
= & \phi\left(\mathrm{K}^{*} \mathrm{~N} 2\right)-\phi\left(\mathrm{K}^{*} \mathrm{~N} 1\right)=\mathrm{TFPV} & & \text { (based on equation 22) }
\end{array}
$$

As a result, a relationship between TFPV and PKP_PE is obtained:

TFPV $=$ K*PKP_PE

This relationship states that the accuracy of PKP (i.e. PKP_PE) is directly controlled by the TFPV, which can only be measured from a baseline test with TPK becoming temporarily available.

Equation (28) also indicates that:

$$
\text { If TFPV }<\delta \text { then PKP_PE }<\delta / K
$$

In other words, the phase error of PKP at sync frequency will be reduced by $K$ times from TFPV at PKP_RF. This property is quite desirable since it will make the requirement for non-stationary PKP application more achievable. One example would be if the PKP-RF happens to sweep through a resonance during a non-stationary time frame causing a 180 degree phase shift in its TFPV. If the harmonic number, $K$, of the PKP_RF is a large number, such 180 degree phase variations will be significantly reduced to $180 / \mathrm{K}$ in its PKP_PE and may still fall within the acceptable range for valid Sync vector phase analysis.

Numerous studies have been performed to measure the actual TFPV of the three SSME pumps (HPFTP, LPFTP; LPOTP) which have TKP (speed probes) available. From these TFPV's, estimates of the PKP-PE can be made to see if acceptable phase errors are achievable. Subsequently, actual PKP_PE can be made and then compared to the estimated PKP_PE from TFPV to validate the accuracy of equation (28) and (29). The results of these studies indicate that the PKP phase error for the HPFTP, LPFTP, and LPOTP of the SSME are all less than 10 degrees and are therefore acceptable for non-stationary rotor-dynamic Sync vector and Bode Diagram analysis. For situations when a true key phasor is not available (e.g. SSME HPOTP), the actual TFPV cannot be measured, and as a result, it 
would be difficult to assess the accuracy of PKP in this case. However, based on the previous studies made with the HPFP, LPFP \& LPOP on the SSME, it would seem reasonable to assume that the PKP phase error for the HPOP should be of the same order.

\section{Analysis of SSME/HPFP Data}

A baseline study was performed to investigate the bearing frequency characteristics of the High Pressure Fuel Turbopump (HPFTP) Turbine End Roller Bearing (TERB) from SSME static-test history which includes 246 SSME ground tests and flights. Figure 11 shows a typical CPLE spectral analysis (CPLE PSD and CPLE-COH) result from SSME ground test 902-861 (HPFTP Unit 8212R1). Since a 4/Rev speed measurement is available for ground testing of this unit, Order Sampling pre-processing was first performed on all accelerometer measurements using the speed probe as the reference signal. Figure-11(a) shows the resulting OS PSD of a Turbine-End accelerometer. (Note: The $\mathrm{x}$-axis of the OS PSD is usually plotted as order frequency. However, the $\mathrm{x}$-axis in figure 11 is plotted as OS frequency which equates to Order Sampling frequency multiplied by a normalized Sync frequency of $512 \mathrm{~Hz}$ in this case). Subsequently, CPLE spectral analysis was performed on the OS signal to generate CPLE-PSD \& CPLE$\mathrm{COH}$ as shown in figure-11(b) and (c). Compared to the OS PSD, the overall noise floor of the CPLE-PSD dropped about $12 \mathrm{~dB}$. As a result, several RPM-related signal components were enhanced (such as sync harmonics at $4 \mathrm{~N}$ and $7 \mathrm{~N}$ ). In particular, a couple of bearing signatures denoted "C" \& "I-2N" also emerge from the noise floor, which represent, respectively, Cage frequency (C) of the TERB and the turbine end roller bearing Inner Roller Pass (IRP) frequency modulated with $2 \mathrm{~N}$ (I-2N). These two bearing signatures are almost unobservable in the OS PSD. In addition, its CPLE-COH delineates meaningful correlation at these two bearing frequencies which further validates that the signal at C and I-2N are indeed RPM-related as a roller bearing signature should be. The presence of these two bearing signals is by no means an indicator of a bearing defect since they can be found in most SSME test accelerometer data (with such OS+CPLE enhancement preformed) and have resulted in no bearing damage. However, their presence provides a valuable window (or media) to monitor bearing conditions; i.e. develop Condition Indicators, by detecting any small frequency shift as bearing wear or defect develops. It should be pointed out that, without OS pre-processing, these bearing frequencies would have been virtually impossible to detect from the raw PSD due to RPM variation.

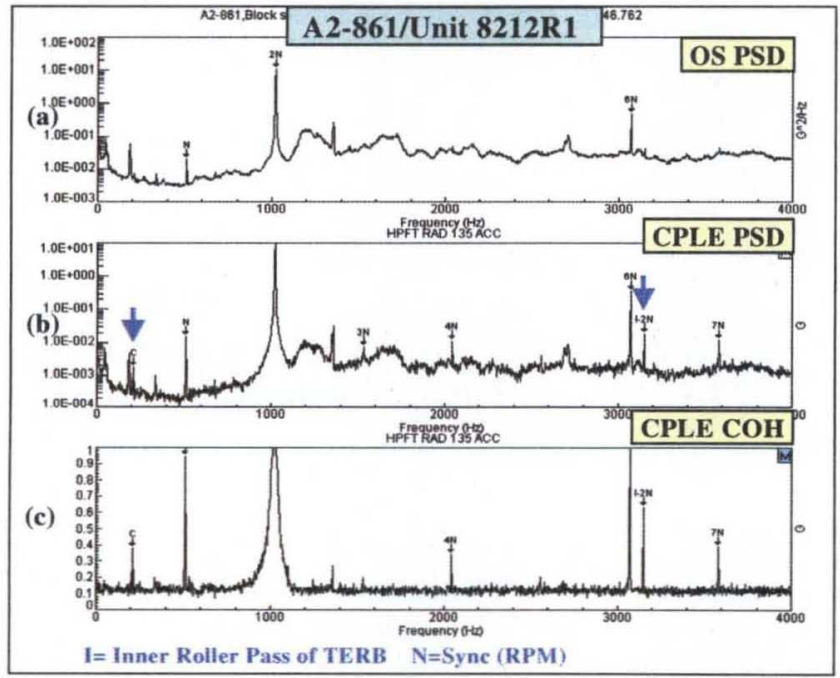

Figure 11. CPLE spectral analysis of a turbine-end accel measurement from SSME ground test A2-861 (HPFTP Unit 8212R1), (a) OS PSD (b) CPLE-PSD (c) CPLE-Coherence

Figure 12(a) shows the same CPLE-PSD from figure 11(b) with frequency zoomed around the I-2N spectral line $(\mathrm{OS}$ Frequency $=3150 \mathrm{~Hz})$. Since the frequency estimation from the FFT-based PSD is limited by its frequency resolution or bandwidth $\Delta$ (which is defined as Sampling Frequency/FFT Block Size or $2.5 \mathrm{~Hz}$ in this example), the actual frequency of I- $2 \mathrm{~N}$ could be anywhere within the frequency band of plus/minus half-bandwidth around the spectral line (i.e. $3150 \pm 0.5 \Delta$ ). Due to the additional signal power decomposition by CPLE Freq analysis into finer CPLE frequency bands at each spectral line, it can further pinpoint the actual frequency within this band and provide 
more accurate frequency estimation as shown in figure 12(b). Figure 12(c) shows the CPLE Frequency spectrogram throughout the entire test period. Its $x$-axis represents the CPLE Frequency band around I-2N spectral line (i.e. $3150 \pm 1.25 \mathrm{~Hz})$. Its $y$-axis represents time. The corresponding RPM profile is shown on the left of the spectrogram. From such CPLE Frequency spectrograms, a more detailed frequency spectrum is obtained which shows the I- $2 \mathrm{~N}$ bearing frequency remaining relatively constant throughout the test. This is an expected normal characteristic since all the roller bearing parameters (such as bearing diameter, pitch diameter and contact angle) remain unchanged. Ninety-eighty percent $(98 \%)$ of the 246 SSME tests analyzed for the baseline study show such characteristics at the I-2N CPLE Frequency. Also of note is that when the RPM drops ( S+250 seconds), the bearing frequency tends to increase slightly. Such a phenomenon is consistently observed in all other SSME tests and could be attributed to a very small bearing geometry change due to load variations.

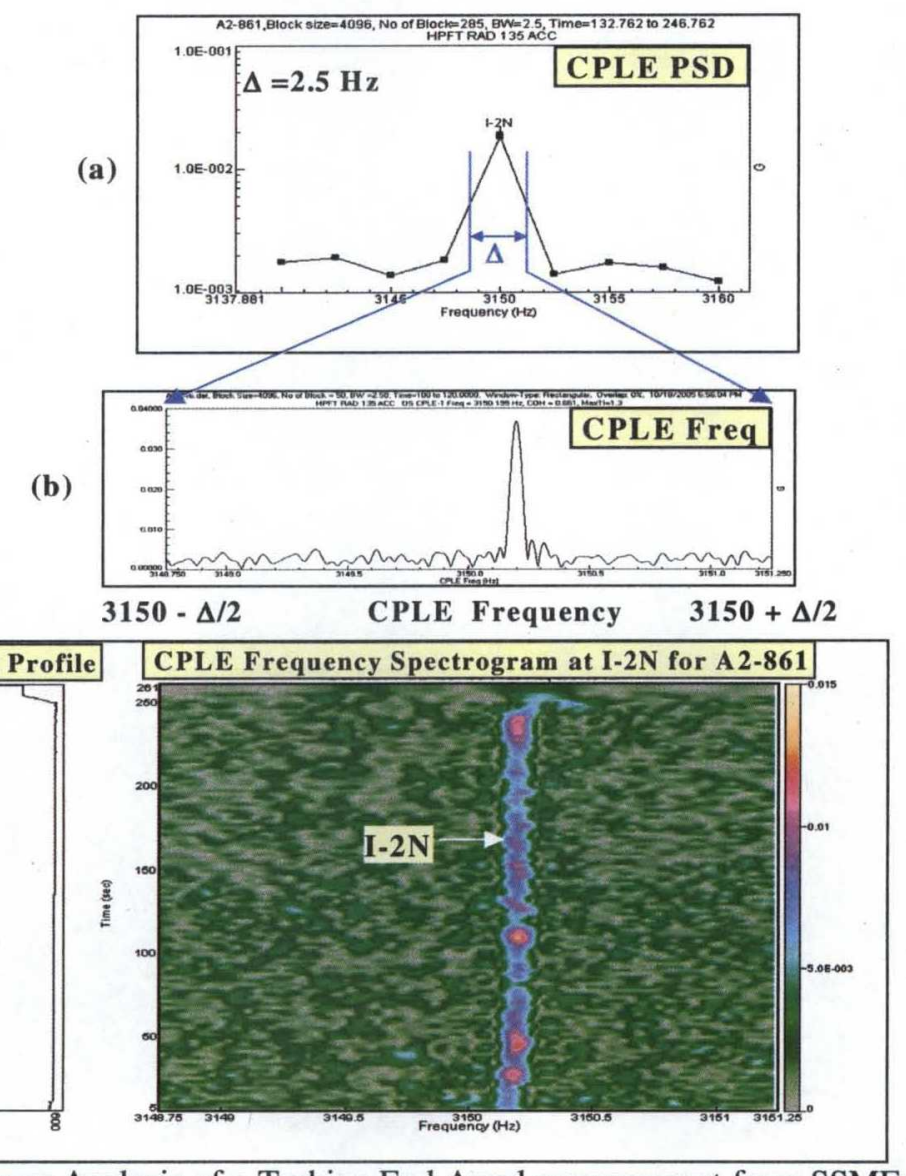

Figure 12. CPLE Frequency Analysis of a Turbine-End Accel measurement from SSME Ground test A2-861 (HPFTP Unit 8212R1).

(a) CPLE-PSD zoomed around I-2N spectral line at $3150 \mathrm{~Hz}$

(b) Signal power decomposes at I-2N into CPLE frequency band.

(c) CPLE Frequency spectrogram at I-2N throughout the entire test period. The $\Delta \mathrm{f}$ delineated on the spectrogram is $2.5 \mathrm{~Hz}$ : i.e. $3151.25-3148.75$.

Figure 13 shows the CPLE-Frequency spectrogram at I-2N from another SSME test, 902-846, with HPFTP Unit 8115R1. Unlike the previous result in Figure 12, an anomalous bearing frequency shift phenomenon is observed. The shift is very small (only about $0.5 \mathrm{~Hz}$ ), but it can be clearly observed by the CPLE Frequency. A TERB bearing cage delamination was found in this turbopump bearing. During post-test disassembly inspections, small amounts of cage material were found to have peeled off the cage. Several other tests of this same unit also exhibited similar I$2 \mathrm{~N}$ bearing frequency shifts. The relationship between such anomalous bearing frequency shifts and the cage delamination mechanism is not clearly understood yet. This example demonstrates the effectiveness of CPLE Frequency analysis in detecting those types of roller bearing defects which can cause small bearing characteristic frequency shifts. 
Figure 14 shows similar analysis results for a recent Space Shuttle Flight, STS-115. The 4/Rev speed measurement for the HPFTP was not available during the flight. Therefore, the PKP technique was used to reconstruct a 1/Rev PKP signal directly from the instantaneous phase signal at $2 \mathrm{~N}$ ( $2^{\text {nd }}$ harmonic of Sync frequency) of an external accelerometer measurement. The resulting PKP signal was then used to perform OS pre-processing followed by CPLE Frequency analysis. Figure 14 shows the resulting CPLE-Frequency spectrogram at I-2N from STS-115 Main Engine 3, HPFTP unit 8118. Much like other nominal units, its I-2N frequency remains relatively constant throughout the flight which is an expected signature characteristic. This example demonstrates that even when a key phasor speed measurement is not available, it is still possible to perform CPLE Frequency analysis as an effective bearing health monitoring tool.

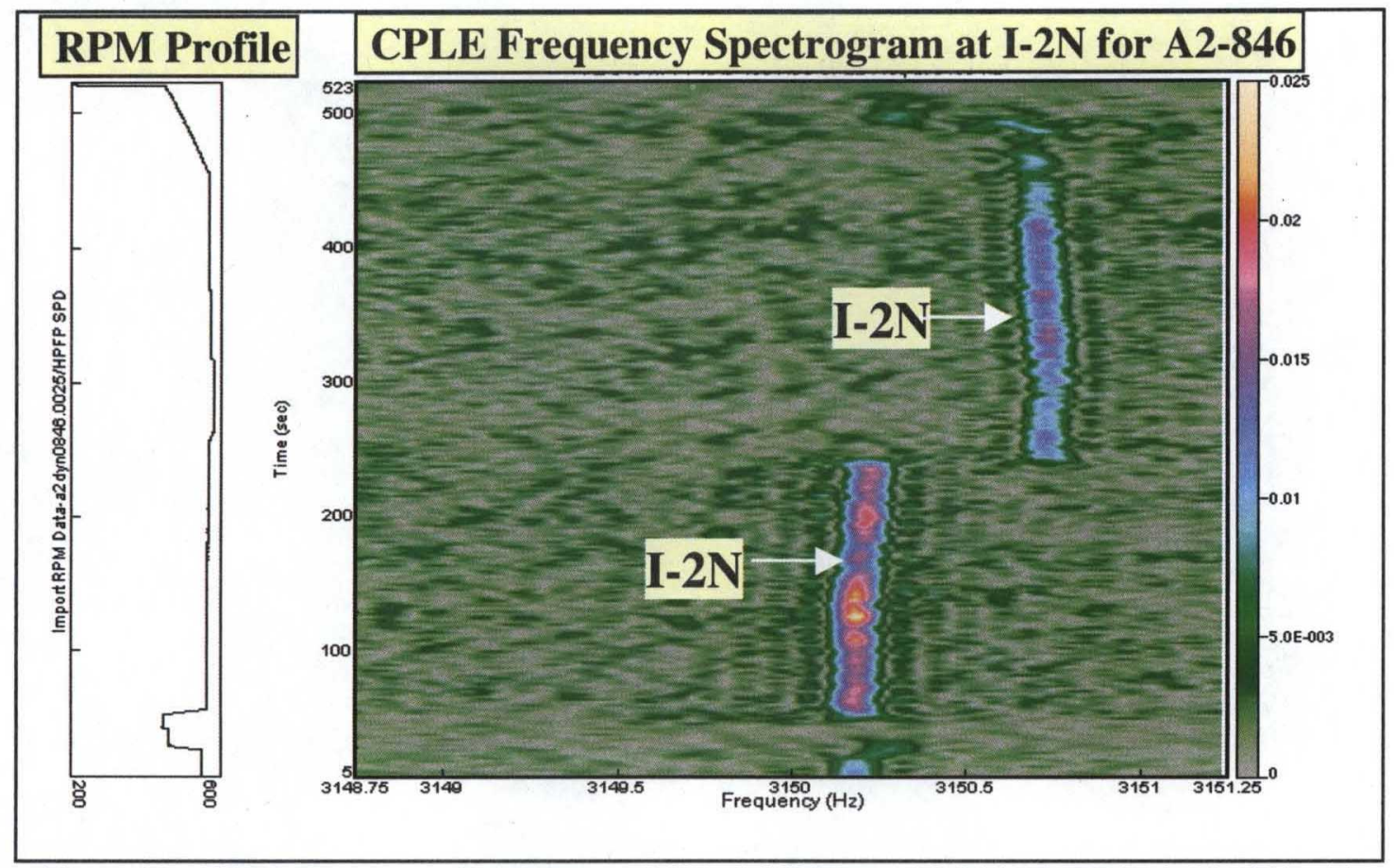

Figure 13. CPLE-Frequency spectrogram at I-2N of SSME test A2-846 HPFTP Unit 8115R1 with TERB bearing cage delamination found.

Since this baseline study was performed, several additional engine static firings and flights have been analyzed using this CPLE Frequency Analysis approach to determine if any other HPFP units exhibited the presence of the I$2 \mathrm{~N}$ frequency shift. To date, this frequency shift has occurred on only three HPFP Units comprising seven static tests. None have been observed to date on Flight Units. The summary of the I- $2 \mathrm{~N}$ frequency shift is shown in Table 2. All HPFTP frequency shifts at $\mathrm{I}-2 \mathrm{~N}$ that have been observed to date have resulted in shifts on the order of $\sim 0.5$ Hz. Only Unit $8115 / R 1$ has had any evidence of cage delamination. Monitoring of this HPFP I-2N frequency shift is continuing. 


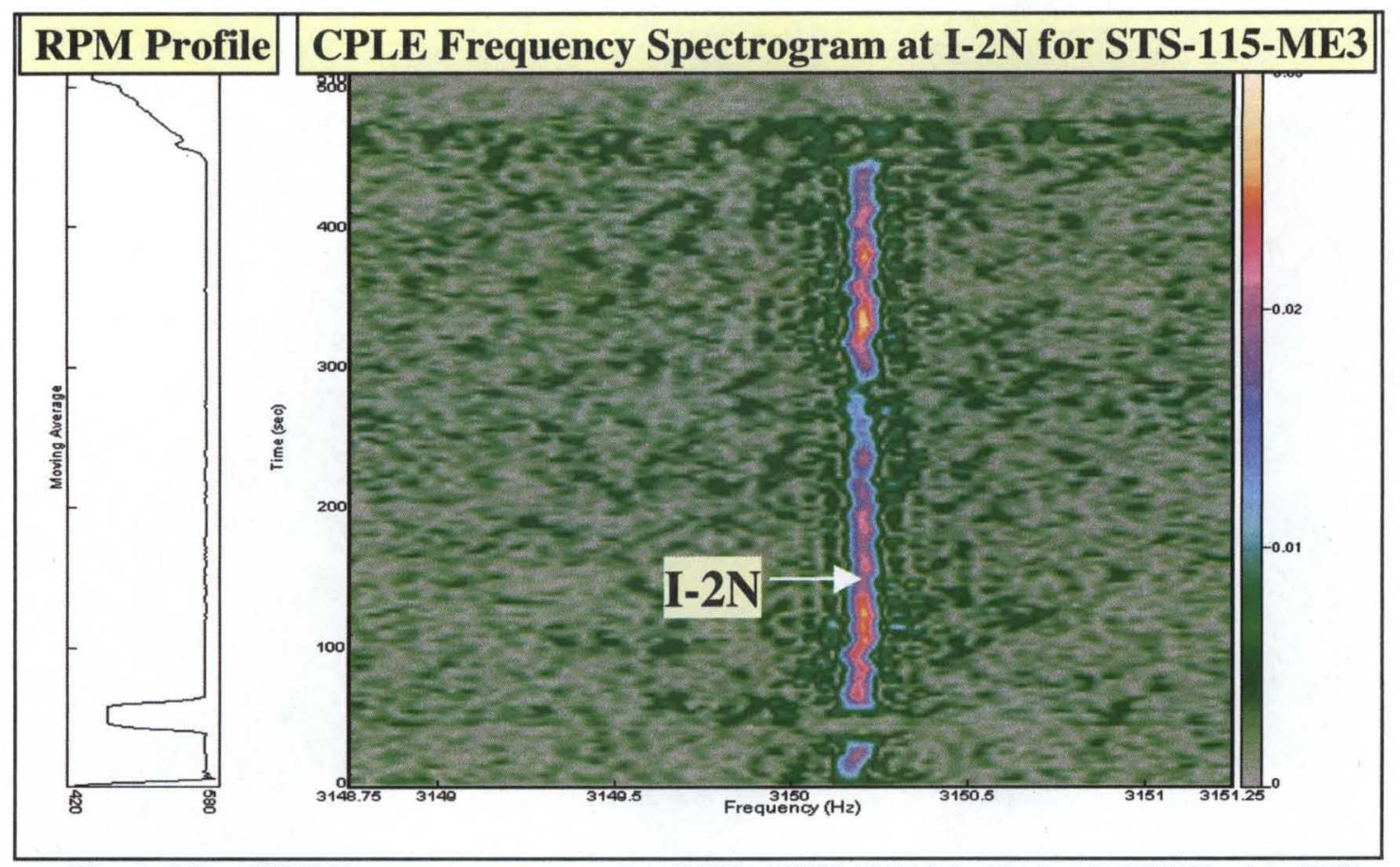

Figure 14. CPLE Frequency spectrogram at I-2N of STS-115 Main Engine 3 HPFTP using PKP to perform OS pre-processing.

\begin{tabular}{|c|c|c|c|}
\hline $\begin{array}{c}\text { HPFP } \\
\text { Unit No. }\end{array}$ & $\begin{array}{c}\text { No. of Tests } \\
\text { On Units }\end{array}$ & $\begin{array}{c}\text { Tests With } \\
\text { Freq. Shift }\end{array}$ & $\begin{array}{c}\text { Frequency Shift Occurred } \\
\text { On These Tests }\end{array}$ \\
\hline 8025 & 1 & 1 & $902-845$ \\
\hline 8112 & 6 & 2 & $902-839,840$ \\
\hline $8115 / R 1$ & 11 & 4 & $902-846,849,850 \& 852$ \\
\hline
\end{tabular}

Table 2. SSME HPFP units with I-2N frequency shifts. Only unit $8115 / R 1$ has the I-2N frequency shift.

\section{Summary/Conclusion}

The CPLE Frequency Analysis approach described herein has identified the presence of a low level bearing signature in the HPFP turbine bearing of the SSME. This signature was observed as a small frequency shift of $\sim 0.5$ $\mathrm{Hz}$ of the IRP turbine end bearing frequency which was being modulated with $2 \mathrm{~N}$; i. e. I- $2 \mathrm{~N}$. This frequency shift was unobservable in the normal vibration spectrum (PSD) and in the Order Synchronization (OS) spectrum. This frequency shift occurs at an OS frequency of $\sim 3150 \mathrm{~Hz}$. The detection of this "frequency shift" has lead to the identification of cage delamination in the HPFP turbine end bearing of one unit.

The CPLE Frequency Analysis approach described herein represents a significant advancement in the diagnostic capability to detect the presence of low level bearing-related signatures in the presence of high-level background noise environments. The SSME has a very high random background noise environment which tends to mask the presence of periodic/quasi-periodic bearing-related frequencies. This has hampered the identification/detection of "incipient" bearing-related signals (signatures) for some time. With the availability of this approach, new insight into the salient characteristics of high-speed rotating machinery bearing signatures in noisy environments can now be gained. This novel CPLE analysis methodology, along with the development of the Pseudo Key Phasor (PKP) (which constitutes the integral parts of the CPLE), will enable vibration engineers to probe deeper into the operational characteristics of roller bearings under very severe operating conditions. 


\section{References}

'Jong J. " Novel Non-Intrusive Vibration Monitoring System for Turbopumps", NASA 2004 SBIR Phase I Final Report, July 2005.

${ }^{2}$ Jong J, Dorland W. " Spectral Enhancement for Incipient Fault Detection of Rotating Machinery", NASA 2000 SBIR Phase II Final Report, July 2004.

${ }^{3}$ Bendat J.S. and Piersol A.G., Engineering Application of Correlation and Spectral Analysis. John Wiley \& Son, 1980.

${ }^{4}$ Jong J., Dorland W., Fiorucci, T. "Coherent Phase Line Enhancer (CPLE), an advanced diagnostics technique", 54th Meeting of the Mechanical Failure Prevention Technology (MFPT). May, 2000.

${ }^{5}$ Jong, J. Jones, J. "Phase Synchronized Enhance-ment Method for Space Shuttle Main Engine Diagnostics", NASA Conference on Advanced Earth-to-Orbit Propulsion Technology, 1994

${ }^{6}$ Freeman, J. A. "Simulating Neural Networks with Mathematica", Addison-Wesley, 1994.

${ }^{7}$ Carpenter, G. A. and Gross, S. "The Art of Adaptive Pattern Recognition by a Self-organizing Neural Network", Computer, 21, 77-88, 1988.

${ }^{8}$ Leon, Robert L. and Martin, J. Dowling. On-line Monitoring of Turbine Blades for Incipient Failure Detection. EPRI Final Report RP1854-1,2, 1986.

${ }^{9}$ Martin, Dowling and Keith, Trainor. "High Frequency Spectral Analysis Study for Helicopter Bearing and Gear Failure Detection.", Liberty Technology Center Final Report for NAVAL Air Development Center. Report No. NADC-90033-60. January 1990.

${ }^{10}$ Jong, J., Jones J., Jones P., Nesman T., Zoladz T., Coffin T., "Nonlinear Correlation Analysis for Rocket Engine Turbomachinery Vibration Diagnostics", 48th Meeting of the Mechanical Failure Prevention Group (MFPG). April, 1994.

${ }^{11}$ Jong, J., Jones, J., McBride J., Coffin, T., "Correlation Identification Between Spectral Components in Turbomachinery Measurements by Generalized Hyper-Coherence", 3rd International Machinery Monitoring And Diagnostic Conference, December, 1991.

${ }^{12}$ Jong J., "Coherence Phase Line Enhancer (CPLE) Spectral Analysis technique" US Patent No.: US 6,408,696 B1, June 25, 2002.

${ }^{13}$ Jong, J., Dorland, W., Fiorucci, T., Zoladz, T., Nesman, T., "Coherent Phase Line Enhancer (CPLE) for Rotating Machinery Diagnostics" AIAA 37th Joint Propulsion Conference, July 2001.

${ }^{14}$ Jong, J., Dorland, W., Fiorucci, T., Zoladz, T., Nesman, T., "Space Shuttle Main Engine Signature Analysis Techniques," Sound \& Vibration, Vol., 34, No. 5, 2000, pp.24-27.

${ }^{15}$ Jong, J., McBride, J., Jones, J., Fiorucci, T., and Zoladz, T., "Synchronous Phase Averaging Method for Machinery Diagnostics," 50th Meeting of the Mechanical Failure Prevention Technology (MFPT) Society. April 1996.

${ }^{16}$ Jong, J., Jones, J., and Zoladz, T., "Phase Synchronized Enhancement Method for Space Shuttle Main Engine Diagnostics," NASA Advanced Earth-to-Orbit Propulsion Technology Conference. 1994.

${ }^{17}$ Vold, H. Herlufsen H. Mains, M Corwin-Renner, D. "Multi Axle Order Tracking with the Vold-Kalman Tracking Filter" Sound \& Vibration, May 1997, Vol 31 No 5.

${ }^{18} \mathrm{McFadden}$, P.D. "Interpolation Techniques for the Time Domain Averaging of Vibration Data With Application to Helicopter Gearbox Monitoring," Aero Propulsion Technical Memorandum 437, AR-004-488, DoD-Science and Technology Org. Aeronautical Research Lab., Commonwealth of Australia, 1986.

${ }^{19}$ Ziemer R. E. and Tranter W. H. Principles of Communications. Houghton Mifflin, Boston, 1985

${ }^{20}$ Bendat J.S. Nonlinear system analysis \& Identification. John Wiley \& Son, New York, 1990 